Synthesis of Benzannulated $N$-heterocycles by a Palladium-Catalyzed C-C/C-N Coupling of Bromoalkylamines

Praew Thansandote, Manuel Raemy, Alena Rudolph, and Mark Lautens*

*mlautens@chem.utoronto.ca

Supporting Information

General. ${ }^{1} \mathrm{H}$ and ${ }^{13} \mathrm{C}$ NMR spectra were recorded in $\mathrm{CDCl}_{3}$ or DMSO with chemical shifts using TMS, residual chloroform or DMSO as internal standard. High-resolution mass spectra were obtained at $70 \mathrm{eV}$. Unless otherwise noted, all materials were obtained from commercial suppliers and used without further purification. Tetrahydrofuran (THF) and diethyl ether $\left(\mathrm{Et}_{2} \mathrm{O}\right)$ were freshly distilled under nitrogen from sodium benzophenone ketyl prior to use. Acetonitrile was distilled from $\mathrm{CaH}_{2}$. In addition, 2-iodo-benzylOTBS-alcohol, ${ }^{1} \quad \mathrm{~N}$-(2-bromoethyl)benzenamine, ${ }^{2}$ and 2-(4-nitrophenylamino)ethanol ${ }^{3}$ were prepared according to literature procedure. Sealed tubes were $0.5 \mathrm{~mL}-2.0 \mathrm{~mL}$ tubes with sealable cap and Teflon septa provided by Biotage. All reactions were performed under nitrogen or argon.

Synthesis of ethyl 2-bromoethylcarbamate (1d): Ethanolamine (5.0 g, 81.9 mmol, 1 equiv), potassium fluoride ( $14.3 \mathrm{~g}, 245.7 \mathrm{mmol}, 3$ equiv), and potassium carbonate (34 g, $245.7 \mathrm{mmol}, 3$ equiv) were dissolved in THF (250 mL) in a $500 \mathrm{~mL}$ round bottom flask. Ethyl chloroformate $(8.22 \mathrm{~mL}, 86.0 \mathrm{mmol}, 1.05$ equiv) was then added dropwise with ice-cooling over a 10 minute period. The resulting mixture was stirred at room temperature for 1 hour and then refluxed overnight. After cooling to room temperature, the mixture was filtered over Celite ${ }^{\circledR}$ and the solid washed with ether $(3 \times 10 \mathrm{~mL})$. The filtrate was concentrated under reduced pressure to reveal ethyl-2-hydroxyethylcarbamate in quantitative yield. The product was identified by comparison with characterization data provided by Hammoch, B.D. et al. ${ }^{4}$ and used without further purification.

Ethyl-2-hydroxyethylcarbamate (12.8 $\mathrm{g}, 96.1 \mathrm{mmol}, 1$ equiv) was dissolved in dichloromethane $(300 \mathrm{~mL})$ in a $500 \mathrm{~mL}$ round bottom flask which was then cooled with ice. Triethylamine (13.4 mL, $96.1 \mathrm{mmol}, 1$ equiv) and then methanesulfonyl chloride (7.44 mL, $96.1 \mathrm{mmol}, 1$ equiv) was added to the ice-cooled bath. The mixture was stirred at $0{ }^{\circ} \mathrm{C}$ until complete (about 10 minutes) and was then cooled to room temperature. The mixture was concentrated under reduced pressure and the resulting residue was dissolved in ethyl acetate and extracted with brine (x2). The organic extracts were combined, dried over $\mathrm{MgSO}_{4}$, and concentrated under reduced pressure to reveal the mesylate of ethyl-2hydroxyethylcarbamate, which was used without further purification. The crude mesylate (18 g, $85.2 \mathrm{mmol}, 1$ equiv) and lithium bromide (20.7 g, $238.6 \mathrm{mmol}, 2.8$ equiv) were dissolved in acetone $(200 \mathrm{~mL})$ in a round bottom flask. The mixture was refluxed

\footnotetext{
${ }^{1}$ Prepared by standard TBS protection of commercially available 2-iodobenzylalcohol.

${ }^{2}$ Prepared by literature procedure according to Kurihara, M. et al. Bioorg. Med. Chem. Lett. 2004, 14, 4131.

${ }^{3}$ Prepared by literature procedure according to Lo Meo, P.; D’Anna, F.; Gruttodauria, M.; Rida, S.; Noto, R. Tetrahedron. 2004, 60, 9099-9111.

${ }^{4}$ Hammoch, B.D. et al. J. Agric. Food Chem. 2002, 50, 29.
} 
overnight. After cooling to room temperature, the mixture was concentrated under reduced pressure and the resulting residue was dissolved in ethyl acetate and extracted with water, $5 \% \mathrm{NaHCO}_{3}$, and brine. The organic extracts were combined, dried over $\mathrm{MgSO}_{4}$, and concentrated under reduced pressure. The crude product was purified by chromatography on silica gel (hexane/EtOAc $=90: 10$ ) to afford ethyl-2bromoethylcarbamate $(13.7 \mathrm{~g}, 82 \%)$ as a clear oil.

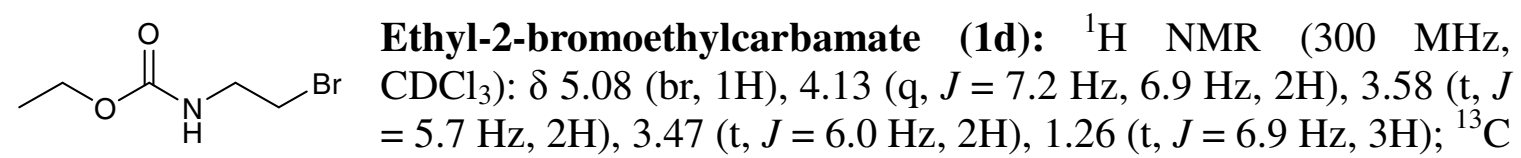

NMR (100 MHz, $\left.\mathrm{CDCl}_{3}\right): \delta 156.7,61.3,42.9,32.8,14.8 ; \mathrm{IR}\left(\mathrm{CDCl}_{3}\right): 3452,3345,2983$, $1715,1520,1235,1057,906,734,649 \mathrm{~cm}^{-1}$; HRMS (ESI) calcd for $\mathrm{C}_{5} \mathrm{H}_{10} \mathrm{BrNO}_{2}$ : 195.9973, found 195.9967.

\section{Synthesis of $N$-4-nitrophenyl-2-bromoalkylamine (1f):}

2-(4-nitrophenylamino)ethanol ${ }^{3}$ (5.0 g, $27.4 \mathrm{mmol}, 1$ equiv), carbon tetrabromide (22.8 g, $68.6 \mathrm{mmol}, 2.5 \mathrm{equiv})$, and triphenylphosphine (10 g, $68.6 \mathrm{mmol}, 2.5$ equiv) was dissolved in THF (150 mL) in a $250 \mathrm{~mL}$ round bottom flask. The mixture was stirred at room temperature until complete. The solvent was removed under reduced pressure and the residue was triturated with hexanes (x5) to remove solid triphenylphosphine oxide. The hexane extracts were combined and the solvent removed under reduced pressure. The resulting residue was purified by chromatography on silica gel (hexane/EtOAc $=50: 50$ ) to afford $N$-(2-bromoethyl)-4-nitrobenzenamine $(2.88 \mathrm{~g}, 43 \%)$ as a bright yellow solid.

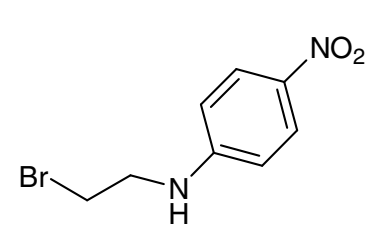

$\boldsymbol{N}$-(2-bromoethyl)-4-nitrobenzenamine (1f). ${ }^{1} \mathrm{H} \quad \mathrm{NMR} \quad(300$ $\left.\mathrm{MHz}, \mathrm{CDCl}_{3}\right): \delta 8.11(\mathrm{~d}, J=8.7 \mathrm{~Hz}, 2 \mathrm{H}), 6.59(\mathrm{~d}, J=9.3 \mathrm{~Hz}$, $2 \mathrm{H}), 4.81(\mathrm{br}, 1 \mathrm{H}), 3.71-3.65(\mathrm{~m}, 2 \mathrm{H}), 3.58(\mathrm{t}, J=4.5 \mathrm{~Hz}, 2 \mathrm{H})$; ${ }^{13} \mathrm{C} \mathrm{NMR}\left(100 \mathrm{MHz}, \mathrm{CDCl}_{3}\right): \delta 152.5,139.0,126.7,111.7,44.8$, 31.0; IR $\left(\mathrm{CDCl}_{3}\right): 1603,1506,1479,1327,1311,1113,906,833$, $729,651 \mathrm{~cm}^{-1}$; HRMS (EI) calcd for $\mathrm{C}_{8} \mathrm{H}_{9} \mathrm{BrN}_{2} \mathrm{O}_{2}: 243.9847$, found 243.9847; mp: 95-97 ${ }^{\circ} \mathrm{C}$.

Synthesis of (2-bromopropyl)-(4-nitrophenyl)-amine (1g): A $100 \mathrm{~mL}$ round-bottom flask, equipped with a stir bar and reflux condenser, was charged with 1-amino-2propanol $(6.2 \mathrm{~mL}, 79 \mathrm{mmol})$ and 1-fluoro-4-nitrobenzene $(7.1 \mathrm{~mL}, 67 \mathrm{mmol}) . \mathrm{CaCO}_{3}$ $(3.3 \mathrm{~g}, 33 \mathrm{mmol})$ was added to the reaction vessel and the mixture was heated to $130{ }^{\circ} \mathrm{C}$ with stirring for $10 \mathrm{hrs}$. The mixture was then allowed to cool to room temperature, diluted with acetone $(25 \mathrm{~mL})$ and $\mathrm{CaCO}_{3}$ was filtered from the reaction mixture. The filtrate was concentrated under reduced pressure and the residue was purified by column chromatography $\left(\mathrm{CH}_{2} \mathrm{Cl}_{2}\right.$ :EtOAc $\left.=3: 1\right)$ to afford 1-(4-nitrophenylamino)-propan-2-ol $(12.7 \mathrm{~g}, 97 \%)$ as a bright yellow solid. ${ }^{1} \mathrm{H}$ NMR (400 MHz, DMSO-d 6 ): $\delta 7.97(\mathrm{~d}, J=9.4$ $\mathrm{Hz}, 2 \mathrm{H}), 7.25(\mathrm{t}, J=5.7 \mathrm{~Hz}, 1 \mathrm{H}), 6.67(\mathrm{~d}, J=9.4 \mathrm{~Hz}, 2 \mathrm{H}), 4.82(\mathrm{~d}, J=4.75 \mathrm{~Hz}, 1 \mathrm{H})$ 3.85-3.77 (m, 1H), 3.15-3.03 (m, 2H), $1.11(\mathrm{~d}, J=6.23 \mathrm{~Hz}, 3 \mathrm{H}) ;{ }^{13} \mathrm{C}$ NMR $(100 \mathrm{MHz}$, DMSO-d ${ }_{6}$ ): $\delta 154.9,135.5,126.2,110.8,64.8,50.2,21.3$; IR (neat): 3356, 2969, 2908, $1601,1505,1471,1307,1111,830,754 \mathrm{~cm}^{-1}$; HRMS calcd for $\mathrm{C}_{9} \mathrm{H}_{13} \mathrm{~N}_{2} \mathrm{O}_{3}$ (ESI) 197.0918, found $197.0920 ; \mathrm{mp}=73-75^{\circ} \mathrm{C}$.

A $250 \mathrm{~mL}$ round-bottom flask equipped with a stir bar, was charged with triphenyl 
phosphine (4.0 g, $15.2 \mathrm{mmol})$ and imidazole $(1.0 \mathrm{~g}, 15.2 \mathrm{mmol})$ in $\mathrm{CH}_{2} \mathrm{Cl}_{2}(50 \mathrm{~mL})$. The solution was cooled to $0^{\circ} \mathrm{C}$ and carbon tetrabromide $(5.0 \mathrm{~g}, 15.2 \mathrm{mmol})$ was added in one portion. The resulting suspension was stirred at $0{ }^{\circ} \mathrm{C}$ for $15 \mathrm{~min}$., at which time a solution of 1-(4-nitrophenylamino)-propan-2-ol (2.0 g, $10.2 \mathrm{mmol})$ in $\mathrm{CH}_{2} \mathrm{Cl}_{2}(50 \mathrm{~mL})$ was added. The reaction mixture was allowed to warm to room temperature and stirring continued for $12 \mathrm{hrs}$. Water $(50 \mathrm{~mL})$ was then added to the reaction mixture and the organic layer was separated. The aqueous layer was further extracted with two portions of $\mathrm{CH}_{2} \mathrm{Cl}_{2}(20$ $\mathrm{mL}$ each). The combined organic layers were dried over $\mathrm{MgSO}_{4}$, filtered and concentrated under reduced pressure. The crude product was purified by column chromatography (Hexanes: $\mathrm{CH}_{2} \mathrm{Cl}_{2}=1: 1-1: 3$ ) to afford (2-bromopropyl)-(4-nitrophenyl)amine (1g) (1.5 g, 58\%) as a bright yellow solid.

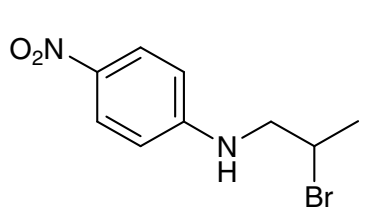

(2-Bromopropyl)-(4-nitrophenyl)-amine (1g): ${ }^{1} \mathrm{H}$ NMR (400 $\left.\mathrm{MHz}, \mathrm{CDCl}_{3}\right): \delta 8.09(\mathrm{~d}, J=9.2 \mathrm{~Hz}, 2 \mathrm{H}), 6.59(\mathrm{~d}, J=9.2 \mathrm{~Hz}, 2 \mathrm{H})$, 4.94 (bs, 1H), 4.34-4.26 (m, 1H), 3.65-3.41 (m, 1H), 1.79 (d, $J=$ $6.7 \mathrm{~Hz}, 3 \mathrm{H}) ;{ }^{13} \mathrm{C} \mathrm{NMR}\left(100 \mathrm{MHz}, \mathrm{CDCl}_{3}\right): \delta 152.7,138.8,126.6$, 111.7, 51.6, 48.4, 23.8; IR (neat): 2976, 2922, 1601, 1528, 1503, 1474, 1305, 1186, 1110, 998, 831, $752 \mathrm{~cm}^{-1}$; HRMS calcd for $\mathrm{C}_{9} \mathrm{H}_{12} \mathrm{BrN}_{2} \mathrm{O}_{2}$ (ESI) 259.0085 , found $259.0076 ; \mathrm{mp}=66-68^{\circ} \mathrm{C}$.

Synthesis of (3-bromopropyl)-phenylamine (1h): A $100 \mathrm{~mL}$ round-bottom flask, equipped with a stir bar and reflux condenser, was charged with a solution of aniline $(0.9$ $\mathrm{mL}, 10 \mathrm{mmol})$ and 1,3-dibromopropane $(6.1 \mathrm{~mL}, 60 \mathrm{mmol})$ in acetonitrile $(20 \mathrm{~mL})$. The solution was heated to reflux for 3 hours. The mixture was then cooled to room temperature, and water $(20 \mathrm{~mL})$ was added. The mixture was extracted with three portions of diethyl ether $(10 \mathrm{~mL})$ and the combined organic layers were dried over $\mathrm{MgSO}_{4}$, filtered and concentrated under reduced pressure. The residue was purified by column chromatography (5\% $\mathrm{Et}_{2} \mathrm{O}$ in hexanes) to afford (3-bromopropyl)-phenylamine (1h) $(627 \mathrm{mg}, 29 \%)$ as a colourless oil.

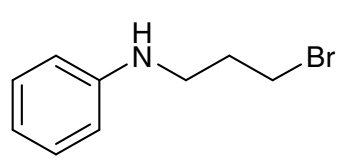

(3-Bromopropyl)-phenylamine (1h): ${ }^{1} \mathrm{H} \mathrm{NMR}\left(400 \mathrm{MHz}, \mathrm{CDCl}_{3}\right)$ : $\delta 7.17(\mathrm{dd}, J=7.4,8.6 \mathrm{~Hz}, 2 \mathrm{H}), 6.71(\mathrm{tt}, J=1.0,7.4 \mathrm{~Hz}, 1 \mathrm{H}), 6.61$ $(\mathrm{dd}, J=1.0,8.6 \mathrm{~Hz}, 2 \mathrm{H}), 3.49(\mathrm{t}, J=6.4 \mathrm{~Hz}, 2 \mathrm{H}), 3.32(\mathrm{t}, J=6.6$ $\mathrm{Hz}, 2 \mathrm{H}), 2.13(\mathrm{tt}, J=6.5 \mathrm{~Hz}, 2 \mathrm{H}) ;{ }^{13} \mathrm{C} \mathrm{NMR}\left(100 \mathrm{MHz}, \mathrm{CDCl}_{3}\right): \delta$ 148.00, 129.5, 117.8, 113.0, 42.2, 32.2, 31.4; IR (neat): 3410, 3044, 3024, 2956, 2935 , 2860, 1602, 1506, 1320, 1254, 749, $692 \mathrm{~cm}^{-1}$; HRMS (EI) calcd for $\mathrm{C}_{9} \mathrm{H}_{12} \mathrm{BrN}$ : 213.0153, found 213.0154 .

General procedure for Sequential Alkylation/Buchwald Hartwig Amination using $N$ (2-bromoethyl)-4-nitrobenzenamine (1f) for the synthesis of 7-methyl-1-(3-methyl-4nitrophenyl)indoline (2f). $N$-4-nitrophenyl-2-bromoethylamine (49 $\mathrm{mg}, 0.2 \mathrm{mmol}$ ), 2iodotoluene $(51 \mu \mathrm{L}, 0.4 \mathrm{mmol}), \mathrm{Pd}(\mathrm{OAc})_{2}(4.5 \mathrm{mg}, 10 \mathrm{~mol} \%)$, TFP $(10.2 \mathrm{mg}, 22 \mathrm{~mol} \%)$, norbornene $\left(38 \mathrm{mg}, 0.6 \mathrm{mmol}\right.$ ), and $\mathrm{Cs}_{2} \mathrm{CO}_{3}(261 \mathrm{mg}, 0.8 \mathrm{mmol})$ was combined in a sealed tube which was then sealed with a Teflon cap. The tube was then purged with nitrogen or argon three times. Dry $\mathrm{CH}_{3} \mathrm{CN}(2.0 \mathrm{~mL})$ was added to the sealed tube and the resulting mixture was stirred at $135{ }^{\circ} \mathrm{C}$ for $20 \mathrm{~h}$. After cooling to room temperature, the mixture was filtered through Celite ${ }^{\circledR}$ and the solvent removed under reduced pressure The 
residue was purified by chromatography on silica gel (hexane/EtOAc $=90: 10$ ) to afford 3a (44 $\mathrm{mg}, 86 \%)$ as a red solid.

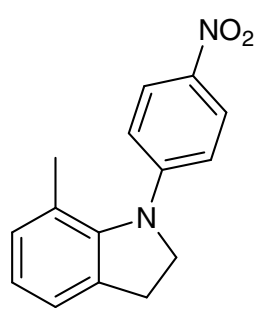

7-methyl-1-(4-nitrophenyl)indoline (2f). ${ }^{1} \mathrm{H}$ NMR $\left(300 \mathrm{MHz}, \mathrm{CDCl}_{3}\right): \delta$ $8.15(\mathrm{~d}, J=9.3 \mathrm{~Hz}, 2 \mathrm{H}), 7.13(\mathrm{~d}, J=6.3 \mathrm{~Hz}, 1 \mathrm{H}), 7.05-6.96(\mathrm{~m}, 2 \mathrm{H})$, $6.81(\mathrm{~d}, J=9.3 \mathrm{~Hz}, 2 \mathrm{H}), 4.18(\mathrm{t}, J=8.1 \mathrm{~Hz}, 2 \mathrm{H}), 3.12(\mathrm{t}, J=8.1 \mathrm{~Hz}, 2 \mathrm{H})$, $2.01(\mathrm{~s}, 3 \mathrm{H}) ;{ }^{13} \mathrm{C}$ NMR $\left(100 \mathrm{MHz}, \mathrm{CDCl}_{3}\right): \delta 192.5,152.6,143.5,134.3$, $130.0,125.2,125.1,123.9,122.7,117.2,56.9,29.9,19.7 ; \mathrm{IR}\left(\mathrm{CDCl}_{3}\right)$ : 1652, 1558, 1471, 1382, 1315, 1113, 911, 730, $651 \mathrm{~cm}^{-1}$; HRMS (ESI) $m / z$ calcd for $\mathrm{C}_{15} \mathrm{H}_{15} \mathrm{~N}_{2} \mathrm{O}_{2}: 255.1134$, found 255.1128 ; mp: $183-185{ }^{\circ} \mathrm{C}$.

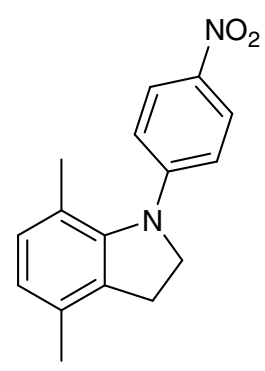

4,7-dimethyl-1-(4-nitrophenyl)indoline (2g). ${ }^{1} \mathrm{H}$ NMR (300 $\mathrm{MHz}$, $\left.\mathrm{CDCl}_{3}\right): \delta 8.14(\mathrm{~d}, J=8.7 \mathrm{~Hz}, 2 \mathrm{H}), 6.95(\mathrm{~d}, J=7.5 \mathrm{~Hz}, 1 \mathrm{H}), 6.84-6.79$ $(\mathrm{m}, 3 \mathrm{H}), 4.18(\mathrm{t}, J=7.5 \mathrm{~Hz}, 2 \mathrm{H}), 3.03(\mathrm{t}, J=8.1 \mathrm{~Hz}, 2 \mathrm{H}), 2.25(\mathrm{~s}, 3 \mathrm{H})$, $1.98(\mathrm{~s}, 3 \mathrm{H}) ;{ }^{13} \mathrm{C} \mathrm{NMR}\left(100 \mathrm{MHz}, \mathrm{CDCl}_{3}\right): \delta 152.7,143.1,140.1$, 132.8, 132.0, 130.0, 125.1, 125.0, 122.3, 117.2, 56.7, 28.6, 19.4, 18.5; IR $\left(\mathrm{CDCl}_{3}\right): 2949,2923,1700,1652,1595,1560,1500,1410,1313$, 1182, 1113, 910, 847, 739, $650 \mathrm{~cm}^{-1}$; HRMS (ESI) $\mathrm{m} / z$ calcd for $\mathrm{C}_{16} \mathrm{H}_{17} \mathrm{~N}_{2} \mathrm{O}_{2}$ : 269.1290, found 269.1284; mp: $125-126{ }^{\circ} \mathrm{C}$.

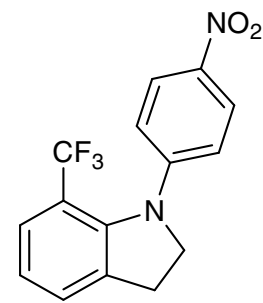

7-(trifluoromethyl)-1-(4-nitrophenyl)indoline (2h). ${ }^{1} \mathrm{H}$ NMR (300 $\left.\mathrm{MHz}, \mathrm{CDCl}_{3}\right): \delta 8.14(\mathrm{~d}, J=9.3 \mathrm{~Hz}, 2 \mathrm{H}), 7.42(\mathrm{~d}, J=8.4 \mathrm{~Hz}, 2 \mathrm{H})$, 7.12-7.05 (m, 3H), $4.16(\mathrm{t}, J=8.1 \mathrm{~Hz}, 2 \mathrm{H}), 3.14(\mathrm{t}, J=7.5 \mathrm{~Hz}, 2 \mathrm{H}) ;{ }^{13} \mathrm{C}$ NMR $\left(75 \mathrm{MHz}, \mathrm{CDCl}_{3}\right): \delta 136.9,128.7,126.7,126.4,126.3,125.2$, 123.4, 119.3, 119.2, 58.1, 29.3; IR $\left(\mathrm{CDCl}_{3}\right): 1652,1558,1506,1471$, 1386, 1097, 907, 734, $651 \mathrm{~cm}^{-1}$; HRMS (ESI) $\mathrm{m} / \mathrm{z}$ calcd for $\mathrm{C}_{15} \mathrm{H}_{12} \mathrm{~F}_{3} \mathrm{~N}_{2} \mathrm{O}_{2}$ : 309.0851, found 309.0845; mp: $120-123{ }^{\circ} \mathrm{C}$.

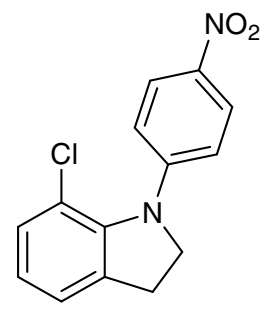

7-chloro-1-(4-nitrophenyl)indoline (2i). ${ }^{1} \mathrm{H}$ NMR $\left(300 \mathrm{MHz}, \mathrm{CDCl}_{3}\right)$ : $\delta 8.17(\mathrm{~d}, J=9.3 \mathrm{~Hz}, 2 \mathrm{H}), 7.21-7.17(\mathrm{~m}, 2 \mathrm{H}), 6.98(\mathrm{~d}, J=7.8 \mathrm{~Hz}, 1 \mathrm{H})$, $6.92(\mathrm{~d}, J=8.7 \mathrm{~Hz} .2 \mathrm{H}), 4.23$ (t, $J=7.8 \mathrm{~Hz}, 2 \mathrm{H}), 3.20$ (t, $J=8.1 \mathrm{~Hz}$, $2 \mathrm{H}) ;{ }^{13} \mathrm{C}$ NMR (100 MHz, $\left.\mathrm{CDCl}_{3}\right): \delta 150.7,136.5,129.3,127.4,126.4$, 124.6, 124.2, 123.5, 120.5, 118.5, 56.9, 29.8; IR $\left(\mathrm{CDCl}_{3}\right): 1588,1558$, 1506, 1471, 1380, 1331, 1096, 903, 738, $651 \mathrm{~cm}^{-1}$; HRMS (EI) $\mathrm{m} / 2$ calcd for $\mathrm{C}_{14} \mathrm{H}_{11} \mathrm{ClN}_{2} \mathrm{O}_{2}: 274.0509$, found 274.0509; mp: 146-148 ${ }^{\circ} \mathrm{C}$.

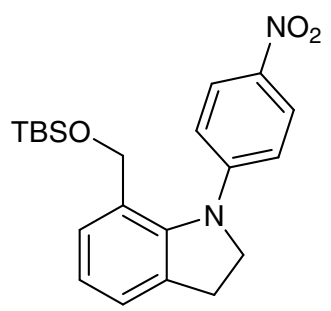

(1-(4-nitrophenyl)indolin-7-yl)-tert-butyldimethylsilylmethanol (2j). ${ }^{1} \mathrm{H}$ NMR $\left(300 \mathrm{MHz}, \mathrm{CDCl}_{3}\right): \delta 8.14(\mathrm{~d}, J=9.3 \mathrm{~Hz}, 2 \mathrm{H}), 7.38(\mathrm{~d}$, $\mathrm{J}=8.1 \mathrm{~Hz}, 1 \mathrm{H}), 7.19(\mathrm{~d}, J=6.9 \mathrm{~Hz}, 1 \mathrm{H}), 7.08(\mathrm{t}, J=7.8 \mathrm{~Hz} .1 \mathrm{H})$, $6.85(\mathrm{~d}, J=8.7 \mathrm{~Hz}, 2 \mathrm{H}), 4.35(\mathrm{~s}, 2 \mathrm{H}), 4.16(\mathrm{t}, J=7.8 \mathrm{~Hz}, 2 \mathrm{H}), 3.12(\mathrm{t}$, $J=8.1 \mathrm{~Hz}, 2 \mathrm{H}), 0.851$ (s, 9H), $-0.0490(\mathrm{~s}, 6 \mathrm{H}) ;{ }^{13} \mathrm{C} \mathrm{NMR}(100 \mathrm{MHz}$, $\mathrm{CDCl}_{3}$ ): 153.1, 141.5, 134.0, 128.6, 126.9, 125.2, 124.0, 123.8, 116.9, 62.0, 57.1, 29.7, 25.8, 18.3, -5.4; IR $\left(\mathrm{CDCl}_{3}\right): 1652,1587,1558$, 
1504, 1471, 1382, 1324, 1316, 1113, 1093, 905, 728, $650 \mathrm{~cm}^{-1}$; HRMS (EI) $\mathrm{m} / z$ calcd for $\mathrm{C}_{21} \mathrm{H}_{28} \mathrm{~N}_{2} \mathrm{O}_{3} \mathrm{Si}: 384.1869$, found $384.1869 ; \mathrm{mp}=$ sublimes at $250{ }^{\circ} \mathrm{C}$.

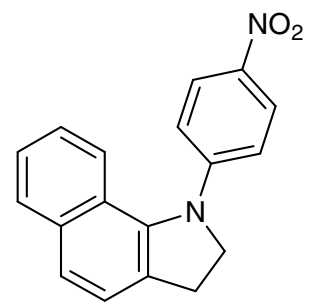

2,3-benzo-1-(4-nitrophenyl)indoline (2k). ${ }^{1} \mathrm{H}$ NMR (300 MHz, $\left.\mathrm{CDCl}_{3}\right): \delta 8.11(\mathrm{~d}, J=9.6 \mathrm{~Hz}, 2 \mathrm{H}), 7.88(\mathrm{~d}, J=7.5 \mathrm{~Hz}, 1 \mathrm{H}), 7.61(\mathrm{~d}$, $J=8.4 \mathrm{~Hz}, 1 \mathrm{H}), 7.46-7.40(\mathrm{~m}, 2 \mathrm{H}), 7.35-7.34(\mathrm{~m}, 2 \mathrm{H}), 6.89(\mathrm{~d}, J=$ $9.6 \mathrm{~Hz}, 2 \mathrm{H}), 4.37(\mathrm{t}, J=8.1 \mathrm{~Hz}, 2 \mathrm{H}), 3.32(\mathrm{t}, J=8.1 \mathrm{~Hz}, 2 \mathrm{H}) ;{ }^{13} \mathrm{C}$ NMR (100 MHz, $\left.\mathrm{CDCl}_{3}\right): \delta 153.3,140.0,134.1,130.9,129.3,125.6$, $125.5,125.2,125.0,124.9,124.2,123.4,123.1,118.0,58.0,30.6$; IR $\left(\mathrm{CDCl}_{3}\right): 1652,1592,1558,1505,1467,1382,1318,1113,1093$, 909, 732, $651 \mathrm{~cm}^{-1}$; HRMS (EI) $\mathrm{m} / z$ calcd for $\mathrm{C}_{18} \mathrm{H}_{14} \mathrm{~N}_{2} \mathrm{O}_{2}: 290.1055$, found 290.1055; mp: $193-195{ }^{\circ} \mathrm{C}$.

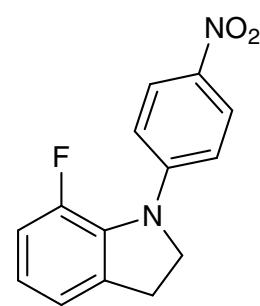

7-fluoro-1-(4-nitrophenyl)indoline (2l). ${ }^{1} \mathrm{H} \mathrm{NMR}\left(400 \mathrm{MHz}, \mathrm{CDCl}_{3}\right)$ : $\delta 8.17(\mathrm{~d}, J=8.8 \mathrm{~Hz}, 2 \mathrm{H}), 7.05(\mathrm{~d}, J=5.6 \mathrm{~Hz}, 1 \mathrm{H}), 7.00-6.91(\mathrm{~m}, 4 \mathrm{H})$, $4.19(\mathrm{t}, J=8.0 \mathrm{~Hz}, 2 \mathrm{H}), 3.24(\mathrm{t}, J=8.8 \mathrm{~Hz}, 2 \mathrm{H}) ;{ }^{13} \mathrm{C} \mathrm{NMR}(75 \mathrm{MHz}$, $\left.\mathrm{CDCl}_{3}\right): \delta 151.7,150.1,148.4,125.2,123.6,123.5,121.2,121.1,116.9$, 116.8, 116.1, 115.9, 55.3, 29.6; IR $\left(\mathrm{CDCl}_{3}\right): 1594,1558,1505,1483$, 1385, 1335, 1316, 1113, 1093, 910, 742, $651 \mathrm{~cm}^{-1}$; HRMS (ESI) $\mathrm{m} / \mathrm{z}$ calcd for $\mathrm{C}_{14} \mathrm{H}_{12} \mathrm{~N}_{2} \mathrm{O}_{2}: 259.0883$, found 259.0877; mp: $133-135{ }^{\circ} \mathrm{C}$.

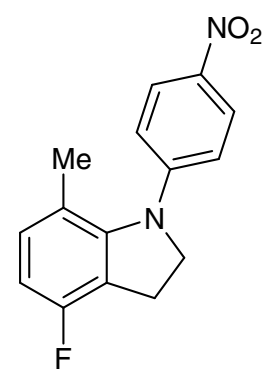

4-fluoro-7-methyl-1-(4-nitrophenyl)indoline (2m). ${ }^{1} \mathrm{H} \quad \mathrm{NMR} \quad(300$ $\left.\mathrm{MHz}, \mathrm{CDCl}_{3}\right): \delta 8.16(\mathrm{~d}, J=9.0 \mathrm{~Hz}, 2 \mathrm{H}), 6.98(\mathrm{t}, J=8.1 \mathrm{~Hz}, 1 \mathrm{H}), 6.83$ $(\mathrm{d}, J=9.3 \mathrm{~Hz}, 2 \mathrm{H}), 6.71(\mathrm{t}, J=8.4 \mathrm{~Hz}, 1 \mathrm{H}), 4.21(\mathrm{t}, J=7.8 \mathrm{~Hz}, 2 \mathrm{H})$, $3.15(\mathrm{t}, J=8.1 \mathrm{~Hz}, 2 \mathrm{H}), 1.96(\mathrm{~s}, 3 \mathrm{H}) ;{ }^{13} \mathrm{C} \mathrm{NMR}\left(100 \mathrm{MHz}, \mathrm{CDCl}_{3}\right)$ : $\delta 192.7,159.3,156.8,152.3,145.9,145.8,141.0,131.8,131.7,125.3$, $120.6,120.5,120.0,119.8,118.0,111.0,110.8,57.5,26.3,26.2,19.3$; IR $\left(\mathrm{CDCl}_{3}\right): 1589,1492,1313,1241,1112,847,796,753 \mathrm{~cm}^{-1}$; HRMS (ESI) $m / z$ calcd for $\mathrm{C}_{15} \mathrm{H}_{13} \mathrm{~N}_{2} \mathrm{O}_{2} \mathrm{~F}: 273.1039$, found 273.1033; mp 140$142{ }^{\circ} \mathrm{C}$.

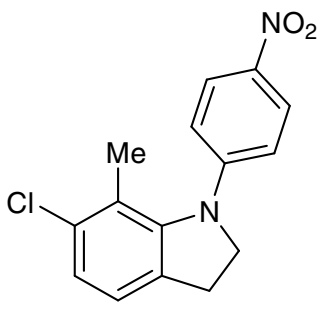

6-chloro-7-methyl-1-(4-nitrophenyl)indoline (2n). ${ }^{1} \mathrm{H}$ NMR (300 $\left.\mathrm{MHz} \mathrm{CDCl}_{3}\right): \delta 8.15(\mathrm{~d}, \mathrm{~J}=9.6 \mathrm{~Hz}, 2 \mathrm{H}), 7.08(\mathrm{q}, \mathrm{J}=8.1 \mathrm{~Hz} \& 5.4$ $\mathrm{Hz}, 2 \mathrm{H}), 6.82(\mathrm{~d}, \mathrm{~J}=9.3 \mathrm{~Hz}, 2 \mathrm{H}), 4.21(\mathrm{t}, \mathrm{J}=8.1 \mathrm{~Hz}, 2 \mathrm{H}), 3.10(\mathrm{t}, \mathrm{J}$ $=8.1 \mathrm{~Hz}, 2 \mathrm{H}), 2.01(\mathrm{~s}, 3 \mathrm{H}) ;{ }^{13} \mathrm{C} \mathrm{NMR}\left(100 \mathrm{MHz}, \mathrm{CDCl}_{3}\right): \delta 152.5$, $145.2,140.7,134.3,132.8,125.2,124.4,123.6,123.1,117.4,57.5$, 29.6, 17.6; IR $\left(\mathrm{CDCl}_{3}\right): 1699,1584,1558,1500,1471,1418,1325$, 1307, 1113, 910, 736, $651 \mathrm{~cm}^{-1}$; HRMS (ESI) $\mathrm{m} / z$ calcd for $\mathrm{C}_{15} \mathrm{H}_{14} \mathrm{ClN}_{2} \mathrm{O}_{2}$ : 289.0744, found 289.0738; mp: 203-205 ${ }^{\circ} \mathrm{C}$.

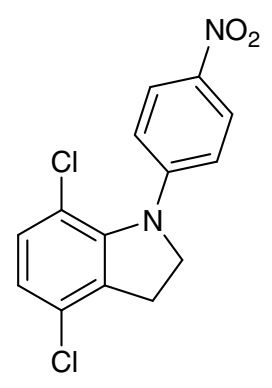

4,7-dichloro-1-(4-nitrophenyl)indoline (2o). ${ }^{1} \mathrm{H} \quad \mathrm{NMR} \quad(300 \mathrm{MHz}$, $\left.\mathrm{CDCl}_{3}\right): \delta 8.18(\mathrm{~d}, J=9.6 \mathrm{~Hz}, 2 \mathrm{H}), 7.13(\mathrm{~d}, J=9.0 \mathrm{~Hz}, 1 \mathrm{H}), 6.95-6.91$ $(\mathrm{m}, 3 \mathrm{H}), 4.25(\mathrm{t}, J=8.4 \mathrm{~Hz}, 2 \mathrm{H}), 3.23(\mathrm{t}, J=8.1 \mathrm{~Hz}, 2 \mathrm{H}) ;{ }^{13} \mathrm{C}$ NMR $(100$ 
$\left.\mathrm{MHz}, \mathrm{CDCl}_{3}\right): \delta 150.3,143.1,141.9,134.5,130.8,129.7,124.7,124.1,119.3,118.3$, 56.7, 29.5; IR $\left(\mathrm{CDCl}_{3}\right): 1601,1584,1558,1505,1455,1422,1330,1311,1217,1144$, 1113, 908, 846, 733, $650 \mathrm{~cm}^{-1}$; HRMS (ESI) $\mathrm{m} / z$ calcd for $\mathrm{C}_{14} \mathrm{H}_{11} \mathrm{Cl}_{2} \mathrm{~N}_{2} \mathrm{O}_{2}: 309.0198$, found 309.0192 ; mp: $135-136^{\circ} \mathrm{C}$.

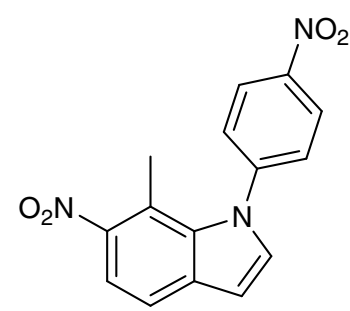

7-methyl-6-nitro-1-(4-nitrophenyl)-1H-indole (3a). ${ }^{1} \mathrm{H} \quad \mathrm{NMR}$ $\left(300 \mathrm{MHz}, \mathrm{CDCl}_{3}\right): \delta 8.41(\mathrm{~d}, J=8.7 \mathrm{~Hz}, 2 \mathrm{H}), 7.82(\mathrm{~d}, J=9.0 \mathrm{~Hz}$, $1 \mathrm{H}), 7.61(\mathrm{~d}, J=8.1 \mathrm{~Hz}, 1 \mathrm{H}), 7.55(\mathrm{~d}, J=8.7 \mathrm{~Hz}, 2 \mathrm{H}), 7.37(\mathrm{~d}, J=$ $3.3 \mathrm{~Hz}, 1 \mathrm{H}), 6.79(\mathrm{~d}, J=3.6 \mathrm{~Hz}, 1 \mathrm{H}), 2.23(\mathrm{~s}, 3 \mathrm{H}) ;{ }^{13} \mathrm{C}$ NMR $(100$ $\left.\mathrm{MHz}, \mathrm{CDCl}_{3}\right): \delta 147.1,146.3,135.3,133.7,127.2,125.5,125.0$, $119.5,119.0,118.5,117.9,105.8,17.2 ; \mathrm{IR}\left(\mathrm{CDCl}_{3}\right): 1652,1598$, 1558, 1527, 1510, 1348, 1337, 1096, 910, 728, $650 \mathrm{~cm}^{-1}$; HRMS (EI) $\mathrm{m} / z$ calcd for $\mathrm{C}_{15} \mathrm{H}_{11} \mathrm{~N}_{3} \mathrm{O}_{4}$ : 297.0750, found 297.0750; mp: $174-176{ }^{\circ} \mathrm{C}$.

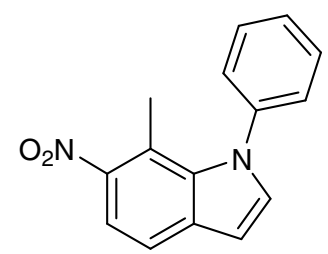

7-methyl-6-nitro-1-phenyl-1H-indole (3b). ${ }^{1} \mathrm{H}$ NMR (400 MHz, $\left.\mathrm{CDCl}_{3}\right): \delta 7.72(\mathrm{~d}, J=8.8 \mathrm{~Hz}, 1 \mathrm{H}), 7.56(\mathrm{~d}, J=9.2 \mathrm{~Hz}, 1 \mathrm{H}), 7.51-$ $7.49(\mathrm{~m}, 3 \mathrm{H}), 7.39-7.37(\mathrm{~m}, 2 \mathrm{H}), 7.34(\mathrm{~d}, J=3.2 \mathrm{~Hz}, 1 \mathrm{H}), 6.69(\mathrm{~d}, J$ $=3.2 \mathrm{~Hz}, 1 \mathrm{H}), 2.17(\mathrm{~s}, 3 \mathrm{H}) ;{ }^{13} \mathrm{C} \mathrm{NMR}\left(100 \mathrm{MHz}, \mathrm{CDCl}_{3}\right)$ : . $\delta 140.9,135.6,135.1,132.6,129.2,128.5,127.3,119.1,118.7,117$. 2, 103.4, 15.8; IR $\left(\mathrm{CDCl}_{3}\right): 1652,1558,1506,1336,909,739,651$ $\mathrm{cm}^{-1}$; HRMS (ESI) $\mathrm{m} / z$ calcd for $\mathrm{C}_{15} \mathrm{H}_{13} \mathrm{~N}_{2} \mathrm{O}_{2}$ : 253.0977, found 253.0971.

General procedure for Sequential Alkylation/Buchwald Hartwig Amination using $\mathbf{N}$ (2-bromoethyl)benzenamine (1e), ethyl-2-bromoethylcarbamate (1d), or (3bromopropyl)-phenylamine (1h) for the synthesis of 7-methyl-1-phenylindoline (2e). $N$-(2-bromoethyl)benzenamine ${ }^{2}$ ( $80 \mathrm{mg}, 0.4 \mathrm{mmol}, 1$ equiv), 2-iodotoluene $(25.5 \mu \mathrm{L}, 0.2$ mmol, 2 equiv), $\mathrm{Pd}(\mathrm{OAc})_{2}(4.5 \mathrm{mg}, 10 \mathrm{~mol} \%)$, TFP (10.2 mg, $\left.22 \mathrm{~mol} \%\right)$, norbornene (38 mg, $0.4 \mathrm{mmol}$ ), and $\mathrm{Cs}_{2} \mathrm{CO}_{3}(521 \mathrm{mg}, 1.6 \mathrm{mmol})$ was combined in a sealed tube which was then sealed with a Teflon cap. The tube was then purged with nitrogen or argon three times. Dry $\mathrm{CH}_{3} \mathrm{CN}(2.0 \mathrm{~mL})$ was added to the sealed tube and the resulting mixture was stirred at $180{ }^{\circ} \mathrm{C}$ for 5 minutes in the microwave. After cooling to room temperature, the mixture was filtered through Celite ${ }^{\circledR}$ and the solvent removed under reduced pressure The residue was purified by chromatography on silica gel (hexane/EtOAc $=90: 10)$ to afford $\mathbf{2 e}(26 \mathrm{mg}, 62 \%)$ as an oil.

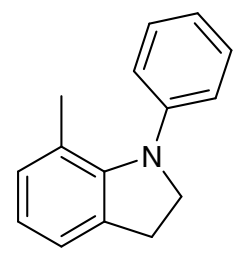

7-methyl-1-phenylindoline (2e). ${ }^{1} \mathrm{H}$ NMR (300 $\left.\mathrm{MHz}, \mathrm{CDCl}_{3}\right): \delta$ 7.29$7.23(\mathrm{~m}, 2 \mathrm{H}), 7.07(\mathrm{~d}, J=6.9 \mathrm{~Hz}, 1 \mathrm{H}), 6.98(\mathrm{~d}, J=7.2 \mathrm{~Hz}, 1 \mathrm{H}), 6.93(\mathrm{~d}, J$ $=8.1 \mathrm{~Hz}, 3 \mathrm{H}), 6.84(\mathrm{t}, J=6.6 \mathrm{~Hz}, 1 \mathrm{H}), 4.01(\mathrm{t}, J=8.1 \mathrm{~Hz}, 2 \mathrm{H}), 3.08(\mathrm{t}, J$ $=8.1 \mathrm{~Hz}, 2 \mathrm{H}), 1.91(\mathrm{~s}, 3 \mathrm{H}) ;{ }^{13} \mathrm{C} \mathrm{NMR}\left(100 \mathrm{MHz}, \mathrm{CDCl}_{3}\right): \delta 148.5,146.5$, 
133.4, 129.7, 128.6, 128.5, 124.0, 122.3, 121.8, 121.5, 120.8, 57.7, 29.7, 19.3; IR (neat): 3034, 2921, 1592, 1495, 1477, 1427, 1361, 1309, 1253, 1211, 1154, 1085, 1043, 756, $727,699 \mathrm{~cm}^{-1}$; HRMS (ESI) $\mathrm{m} / z$ calcd for $\mathrm{C}_{15} \mathrm{H}_{15} \mathrm{~N}$ : 209.1205; found 209.1205.

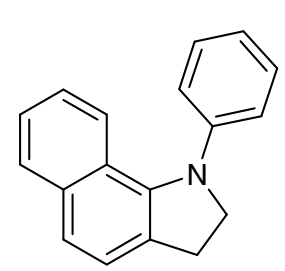

2,3-benzo-1-phenylindoline (2s): ${ }^{1} \mathrm{H}-\mathrm{NMR}\left(300 \mathrm{MHz}, \mathrm{CDCl}_{3}\right) \delta 7.81$ $(\mathrm{d}, J=8.8 \mathrm{~Hz}, 1 \mathrm{H}), 7.66(\mathrm{~d}, J=2.0 \mathrm{~Hz}, 1 \mathrm{H}), 7.46(\mathrm{~d}, J=8.0 \mathrm{~Hz}, 1 \mathrm{H})$, $7.40(\mathrm{~d}, J=8.4 \mathrm{~Hz}, 1 \mathrm{H}), 7.37-7.32(\mathrm{~m}, 1 \mathrm{H}), 7.45-7.18(\mathrm{~m}, 2 \mathrm{H}), 6.99(\mathrm{~d}$, $\mathrm{J}=8.4 \mathrm{~Hz}, 2 \mathrm{H}), 6.80-6.79(\mathrm{~m}, 1 \mathrm{H}), 6.42-6.40(\mathrm{~m}, 1 \mathrm{H}), 4.20(\mathrm{t}, J=8.0$ $\mathrm{Hz}, 2 \mathrm{H}), 3.27(\mathrm{t}, \mathrm{J}=8.8 \mathrm{~Hz}, 2 \mathrm{H}) ;{ }^{13} \mathrm{C}-\mathrm{NMR}\left(75 \mathrm{MHz}, \mathrm{CDCl}_{3}\right) \delta 147.8$, $147.7,134.2,129.0,128.8,125.1,124.8,124.5,123.4,123.1,122.5$, 121.5, 121.3, 121.2, 111.1, 110.9, 58.9, 30.3; IR( $\left(\mathrm{CDCl}_{3}\right): 1651,1557,1504,1489,1383$, 1093, 909, $737 \mathrm{~cm}^{-1}$; HRMS (ESI) calc. for $\mathrm{C}_{18} \mathrm{H}_{15} \mathrm{~N} 245.1205$; found 245.1206.

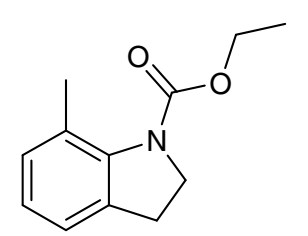

Ethyl 7-methylindoline-1-carboxylate (2d): ${ }^{1} \mathrm{H}-\mathrm{NMR} \quad(300 \mathrm{MHz}$, $\left.\mathrm{CDCl}_{3}\right) \delta ; 7.05-6.95(\mathrm{~m}, 3 \mathrm{H}), 4.24(\mathrm{q}, J=6.9 \mathrm{~Hz} \& 7.2 \mathrm{~Hz}, 2 \mathrm{H}), 4.11$ $(\mathrm{t}, J=7.8 \mathrm{~Hz}, 2 \mathrm{H}), 2.99(\mathrm{t}, J=7.5 \mathrm{~Hz}, 2 \mathrm{H}), 2.30(\mathrm{~s}, 3 \mathrm{H}), 1.33(\mathrm{t}, J=$ $7.2 \quad \mathrm{~Hz}, \quad 3 \mathrm{H}) ;{ }^{13} \mathrm{C}-\mathrm{NMR} \quad\left(100 \quad \mathrm{MHz}, \quad \mathrm{CDCl}_{3}\right)$ $\delta 154.8,141.6,134.3,129.9,128.0,124.5,121.8,61.8,50.8,29.6,20.2$ , 14.6; IR(CDCl $\left.{ }_{3}\right): 2922,1700,1653,1558,1465,1375,1332,1237,1188,1110,1052$ $\mathrm{cm}^{-1}$; HRMS (ESI) calc. for $\mathrm{C}_{12} \mathrm{H}_{15} \mathrm{NO}_{2}$ : 206.1181; found 206.1175.

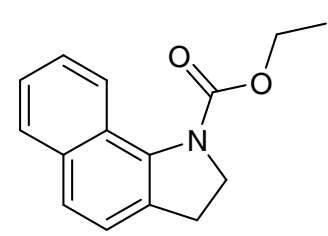

Ethyl 6,7-benzoindoline-1-carboxylate (2r): ${ }^{1} \mathrm{H}-\mathrm{NMR}(400 \mathrm{MHz}$, $\left.\mathrm{CDCl}_{3}\right) \delta 7.99(\mathrm{~d}, J=8.8 \mathrm{~Hz}, 1 \mathrm{H}), 7.81(\mathrm{~d}, J=8.4 \mathrm{~Hz}, 1 \mathrm{H}), 7.61(\mathrm{~d}$, $J=8.4 \mathrm{~Hz}, 1 \mathrm{H}), 7.48-7.38(\mathrm{~m}, 2 \mathrm{H}), 7.35(\mathrm{~d}, J=8.4 \mathrm{~Hz}, 1 \mathrm{H}), 4.33-$ $4.26(\mathrm{~m}, 4 \mathrm{H}), 3.17(\mathrm{t}, J=7.6 \mathrm{~Hz}, 2 \mathrm{H}), 1.32(\mathrm{t}, J=7.2 \mathrm{~Hz}, 3 \mathrm{H}) ;{ }^{13} \mathrm{C}-$ NMR $\left(100 \mathrm{MHz}, \mathrm{CDCl}_{3}\right) \delta 138.5,133.8,130.6,128.1,125.8,125.4$, $125.1,124.9,124.5,122.4,62.0,51.5,30.1,14.6 ; \mathrm{IR}\left(\mathrm{CDCl}_{3}\right): 2982,1699,1574,1520$, 1464, 1406, 1377, 1347, 1329, 1237, 1187, 1100, 1033, 909, 732, $651 \mathrm{~cm}^{-1}$; HRMS (ESI) calc. for $\mathrm{C}_{15} \mathrm{H}_{15} \mathrm{NO}_{2}$ : 242.1181; found 242.1175.

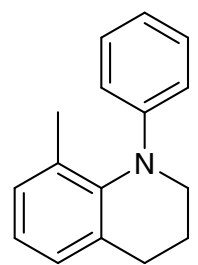

8-Methyl-1-phenyl-1,2,3,4-tetrahydroquinoline (5a): ${ }^{1} \mathrm{H}$ NMR $(400 \mathrm{MHz}$, $\left.\mathrm{CDCl}_{3}\right): \delta 7.19(\mathrm{~m}, 2 \mathrm{H}), 7.02-6.92(\mathrm{~m}, 3 \mathrm{H}), 6.87(\mathrm{tt}, J=1.1,7.5 \mathrm{~Hz}, 1 \mathrm{H})$, 6.81-6.78 (m, 2H), 3.71-3.68 (m, 2H), $2.80(\mathrm{t}, J=6.8 \mathrm{~Hz}, 2 \mathrm{H}), 1.87-1.81(\mathrm{~m}$, $2 \mathrm{H}), 1.86(\mathrm{~s}, 3 \mathrm{H}) ;{ }^{13} \mathrm{C} \mathrm{NMR}\left(100 \mathrm{MHz}, \mathrm{CDCl}_{3}\right): \delta 150.7,142.7,132.8$, 132.0, 129.1, 128.9, 127.2, 123.0, 120.79, 120.75, 120.67, 52.2, 27.7, 21.7, 19.2; IR (neat): 3031, 2937, 2860, 1589, 1491, 1465, 756, $697 \mathrm{~cm}^{-1}$; HRMS (EI) $m / z$ calcd for $\mathrm{C}_{16} \mathrm{H}_{17} \mathrm{~N}: 223.1361$; found 223.1364 .

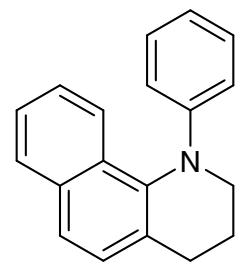

1-Phenyl-1,2,3,4-tetrahydrobenzo[h]quinoline (5b): ${ }^{1} \mathrm{H}$ NMR (400 $\left.\left.\mathrm{MHz}_{\mathrm{CDCl}}\right)\right): \delta 7.74(\mathrm{~d}, J=8.1 \mathrm{~Hz}, 1 \mathrm{H}), 7.64(\mathrm{~d}, J=8.6 \mathrm{~Hz}, 1 \mathrm{H}), 7.52$ $(\mathrm{d}, J=8.4 \mathrm{~Hz}, 1 \mathrm{H}), 7.31(\mathrm{t}, J=7.3 \mathrm{~Hz}, 1 \mathrm{H}), 7.26-7.13(\mathrm{~m}, 4 \mathrm{H}), 6.89-6.82$ $(\mathrm{m}, 3 \mathrm{H}), 3.85-3.82(\mathrm{~m}, 2 \mathrm{H}), 2.97(\mathrm{t}, J=6.8 \mathrm{~Hz}, 1 \mathrm{H}), 1.90(\mathrm{~m}, 2 \mathrm{H}) ;{ }^{13} \mathrm{C}$ NMR $\left(100 \mathrm{MHz}, \mathrm{CDCl}_{3}\right): \delta 151.9,139.5,133.5,129.1,129.0,128.29$, 
128.26, 127.8, 125.4, 125.1, 124.8, 123.8, 122.0, 121.2, 52.8, 27.8, 20.6; IR (neat): 3050, 2931, 2874, 1597, 1569, 1490, 1394, 803, 780, 758, $697 \mathrm{~cm}^{-1}$; HRMS (EI) $\mathrm{m} / z$ calcd for $\mathrm{C}_{19} \mathrm{H}_{17} \mathrm{~N}: 259.1361$; found 259.1362.

Synthesis of 3,7-dimethyl-1-(4-nitrophenyl)-2,3-dihydro-1H-indole (4a): (2Bromopropyl)-(4-nitrophenyl)-amine (1g) $(52 \mathrm{mg}, 0.2 \mathrm{mmol}), 2$-iodotoluene $(51 \mu \mathrm{L}, 0.4$ mmol), $\mathrm{Pd}(\mathrm{OAc})_{2}(4.5 \mathrm{mg}, 10 \mathrm{~mol} \mathrm{\%})$, tri-p-tolylphosphine (13.4 mg, $\left.22 \mathrm{~mol} \%\right)$, norbornene $(38 \mathrm{mg}, 0.6 \mathrm{mmol})$, and $\mathrm{Cs}_{2} \mathrm{CO}_{3}(261 \mathrm{mg}, 0.8 \mathrm{mmol})$ were combined in a sealed tube which was then sealed with a Teflon cap. The tube was then purged with nitrogen or argon three times. Dry DMF $(2.0 \mathrm{~mL})$ was added to the sealed tube and the resulting mixture was stirred at $135{ }^{\circ} \mathrm{C}$ for $16 \mathrm{~h}$. After cooling to room temperature, the mixture was diluted with diethyl ether $(5 \mathrm{~mL})$ and washed with water $(5 \mathrm{~mL})$. The aqueous phase was extracted with two additional portions of ether ( $5 \mathrm{~mL}$ each) and the combined organic layers were dried over $\mathrm{MgSO}_{4}$, filtered and concentrated. The crude product was purified by column chromatography (5-20\% $\mathrm{Et}_{2} \mathrm{O}$ in hexanes) to afford 3,7dimethyl-1-(4-nitrophenyl)-2,3-dihydro- $1 H$-indole (4a) $(30 \mathrm{mg}, 55 \%)$ as a light orange solid.

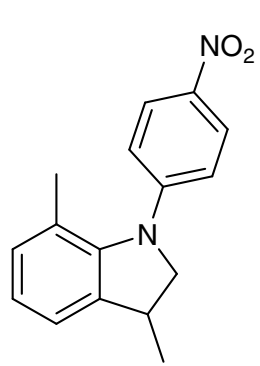

3,7-Dimethyl-1-(4-nitrophenyl)-2,3-dihydro-1H-indole (4a): ${ }^{1} \mathrm{H}$ NMR $\left(400 \mathrm{MHz}, \mathrm{CDCl}_{3}\right): \delta 8.13(\mathrm{~d}, J=9.2 \mathrm{~Hz}, 2 \mathrm{H}), 7.13(\mathrm{~d}, J=7.0 \mathrm{~Hz}, 1 \mathrm{H})$, 7.06-6.99 (m, 2H), $6.81(\mathrm{~d}, J=9.2 \mathrm{~Hz}, 2 \mathrm{H}), 4.37-4.30(\mathrm{~m}, 1 \mathrm{H}), 3.52$ (dd, $J=15.4 \mathrm{~Hz}, 8.2 \mathrm{~Hz}, 1 \mathrm{H}), 2.52(\mathrm{~d}, J=15.4 \mathrm{~Hz}, 1 \mathrm{H}), 2.01(\mathrm{~s}, 3 \mathrm{H}), 1.42$ (d, $J=6.6 \mathrm{~Hz}, \quad 3 \mathrm{H}) ;{ }^{13} \mathrm{C} \quad \mathrm{NMR} \quad\left(100 \quad \mathrm{MHz}, \mathrm{CDCl}_{3}\right)$ : $\delta 152.6,142.4,140.5,133.4,130.1,126.2,125.4,124.3,123.4,116.5,65$ $.0,37.7,22.5,19.7$; IR (neat): 2969, 2915, 1585, 1497, 1318, 1266, 1184, 1111, 1051, 843, 764, 752, $697 \mathrm{~cm}^{-1}$; HRMS (ESI) $\mathrm{m} / \mathrm{z}$ calcd for $\mathrm{C}_{16} \mathrm{H}_{17} \mathrm{~N}_{2} \mathrm{O}_{2}$ (ESI) 269.1296, found 269.1284; mp $=116-119{ }^{\circ} \mathrm{C}$.<smiles>CCOC(=O)NCCBr</smiles> 

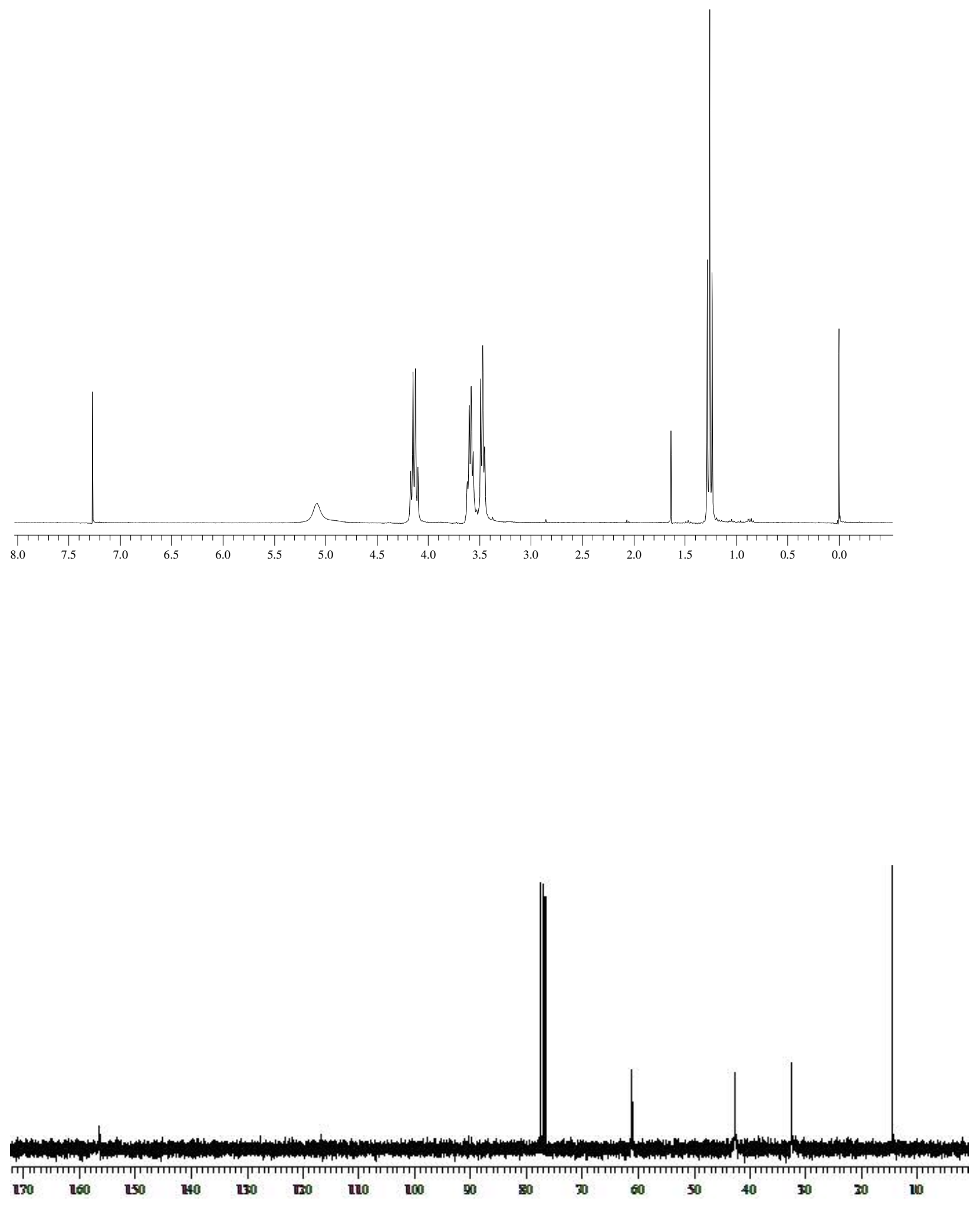

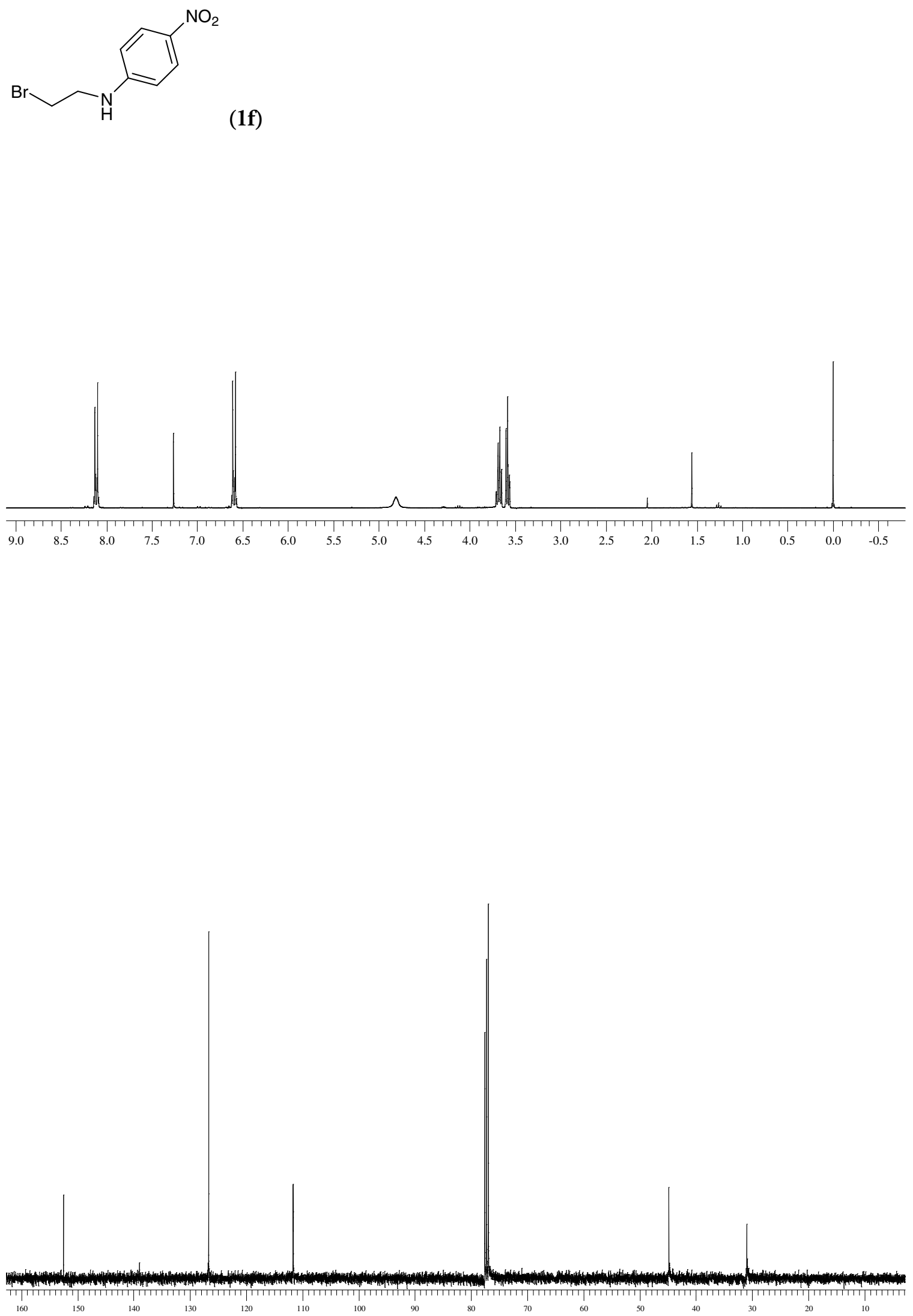

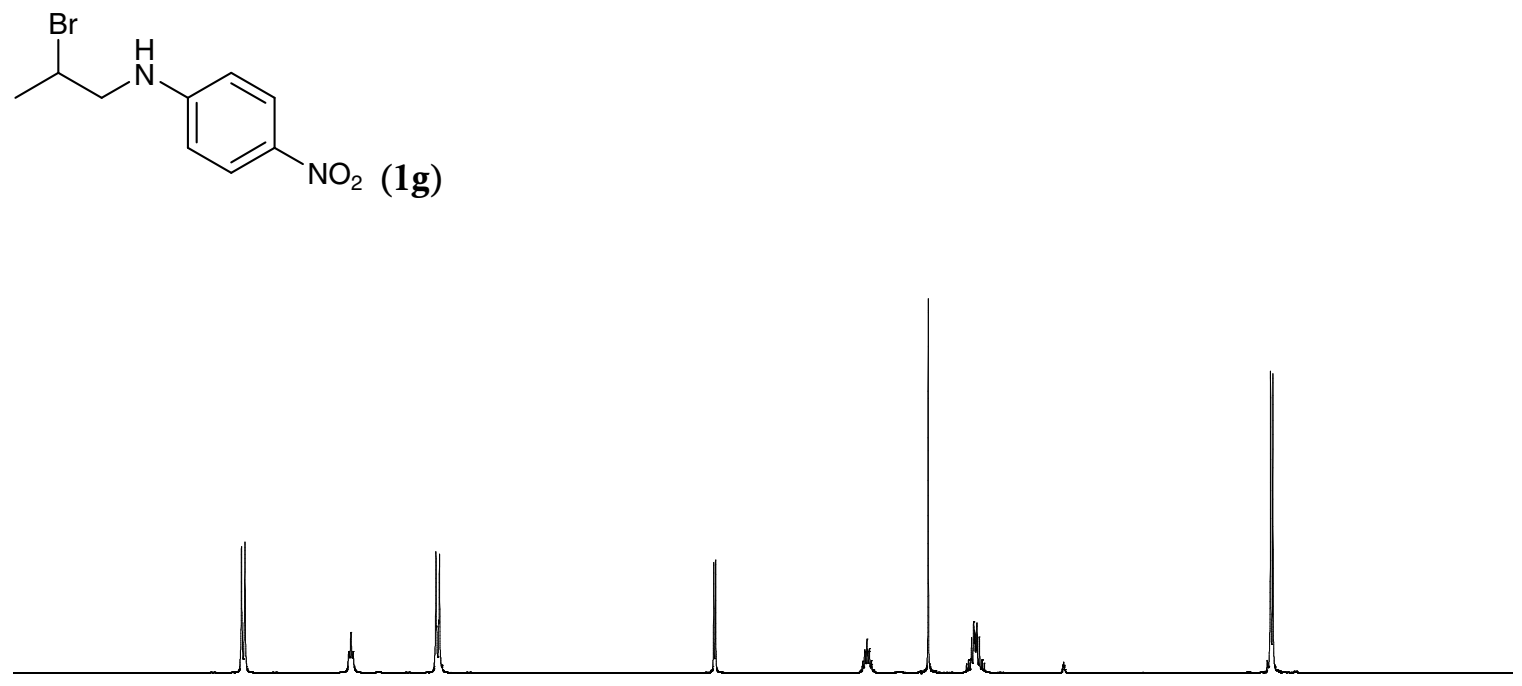

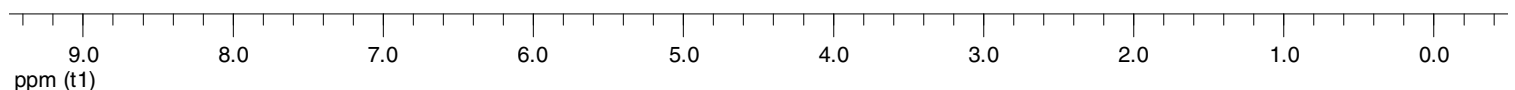

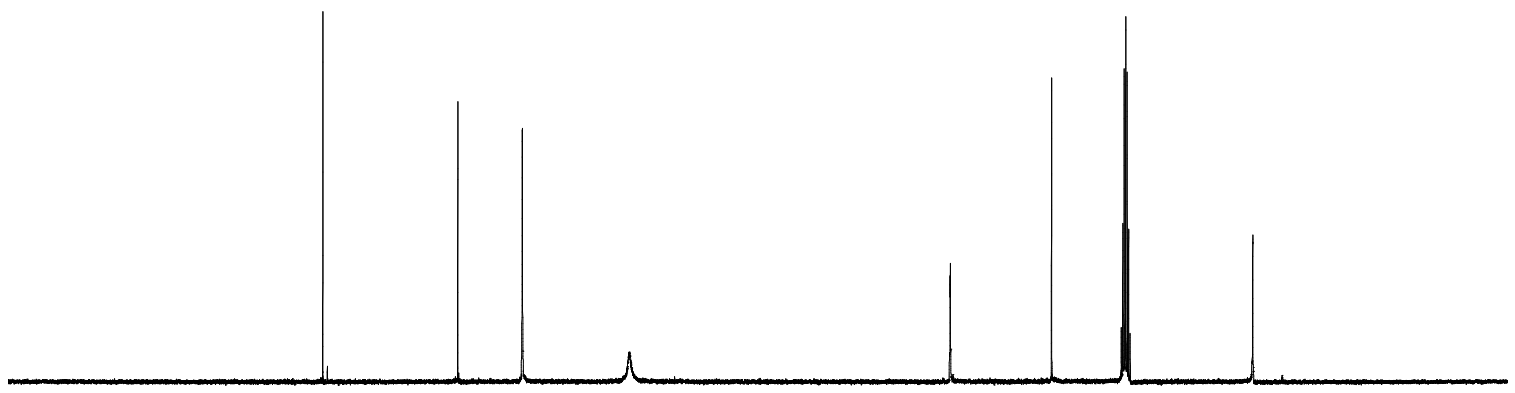

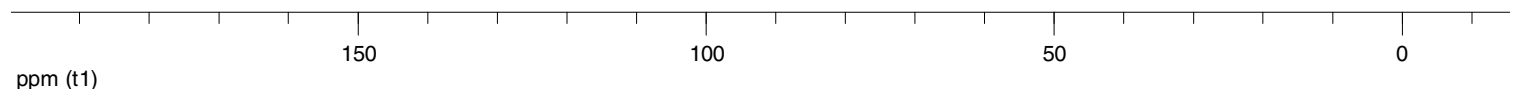



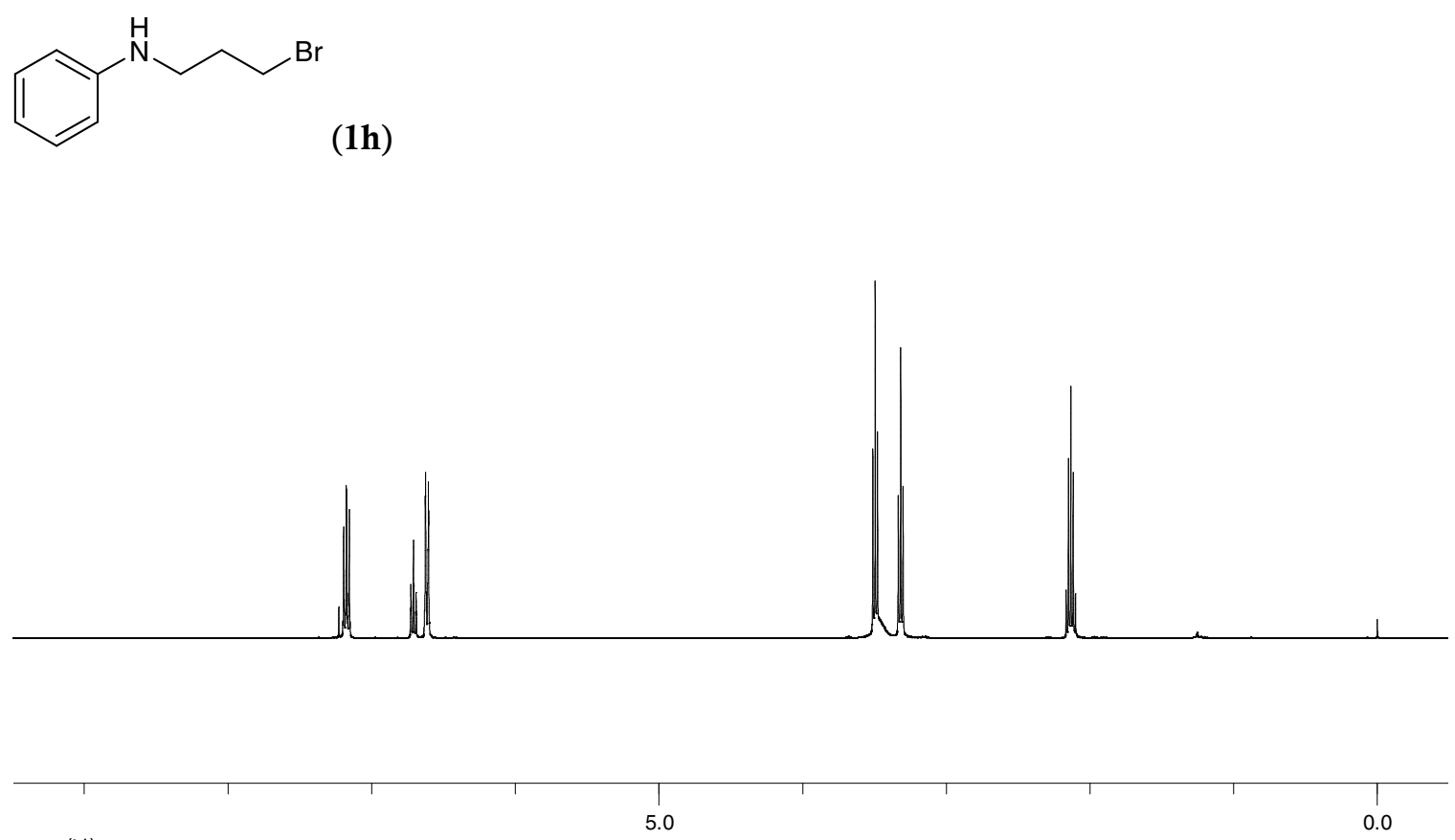

ppm (t1)

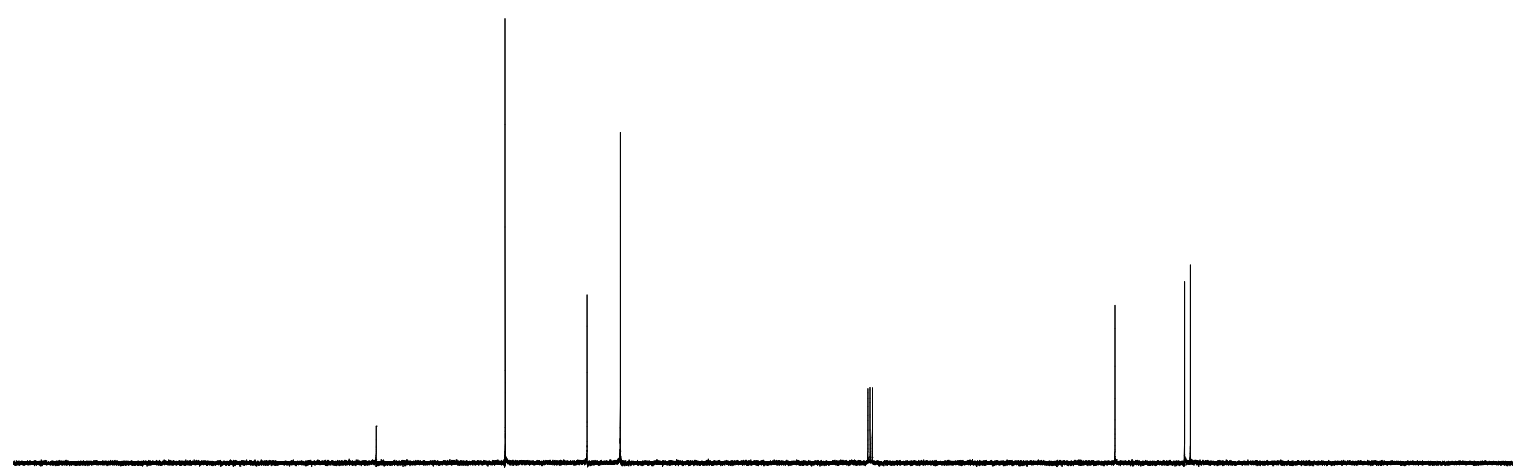

ppm (t1)

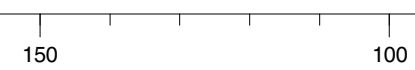

50 
13

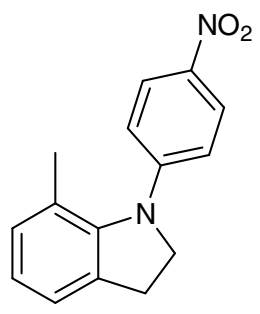

(2f)
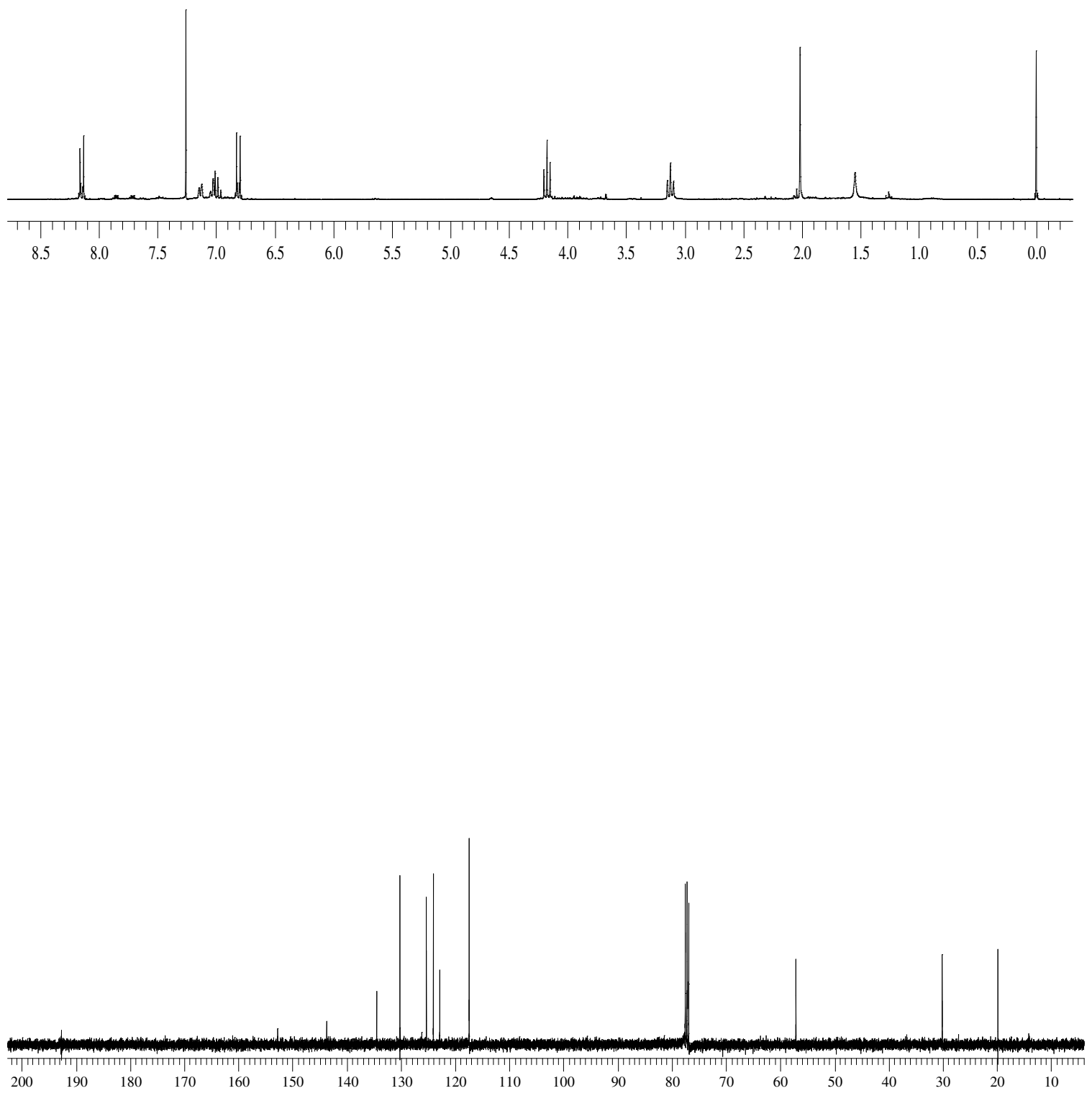


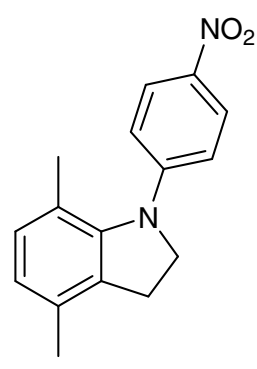

(2g)
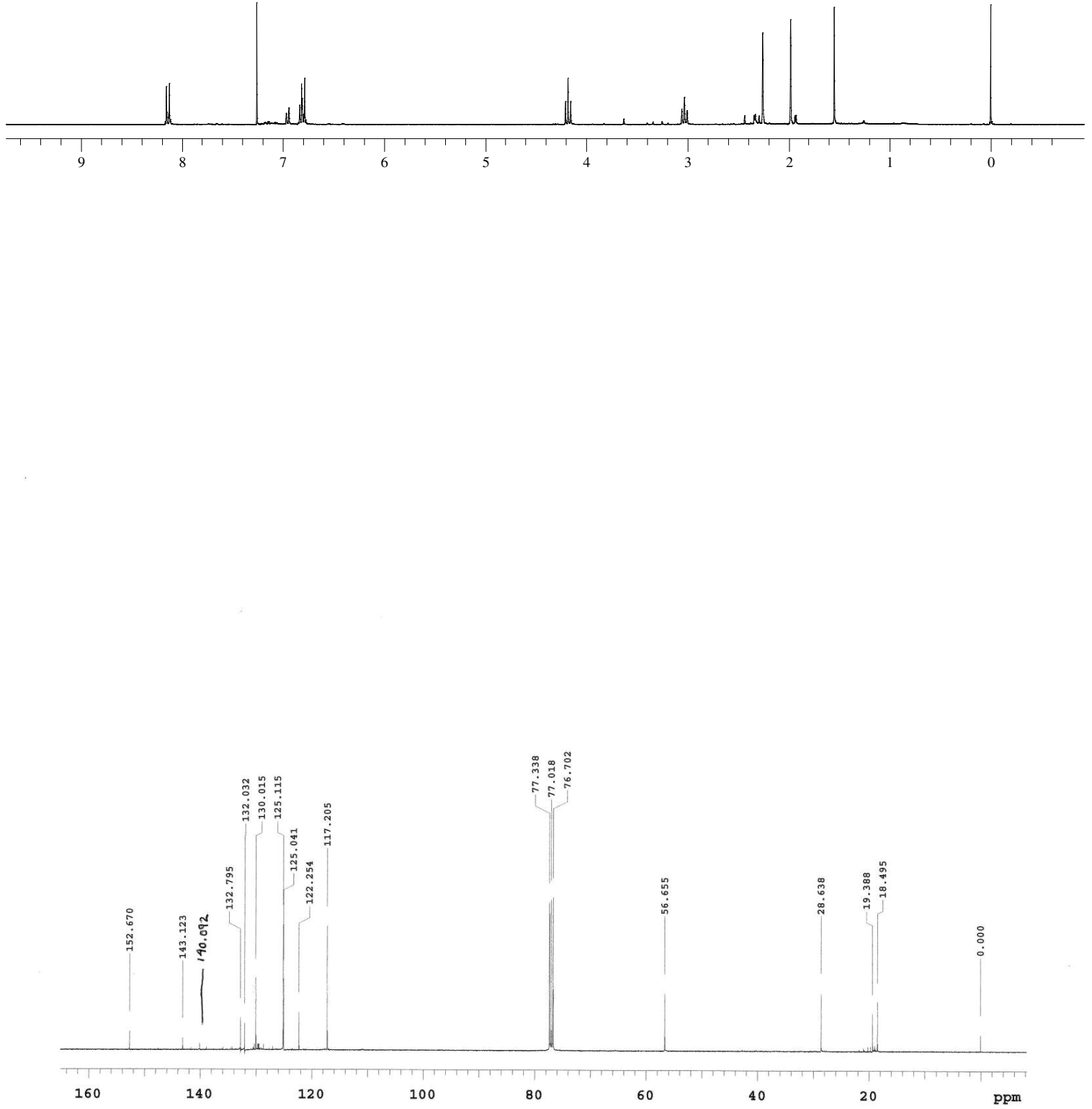


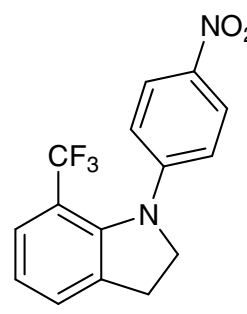

(2h)
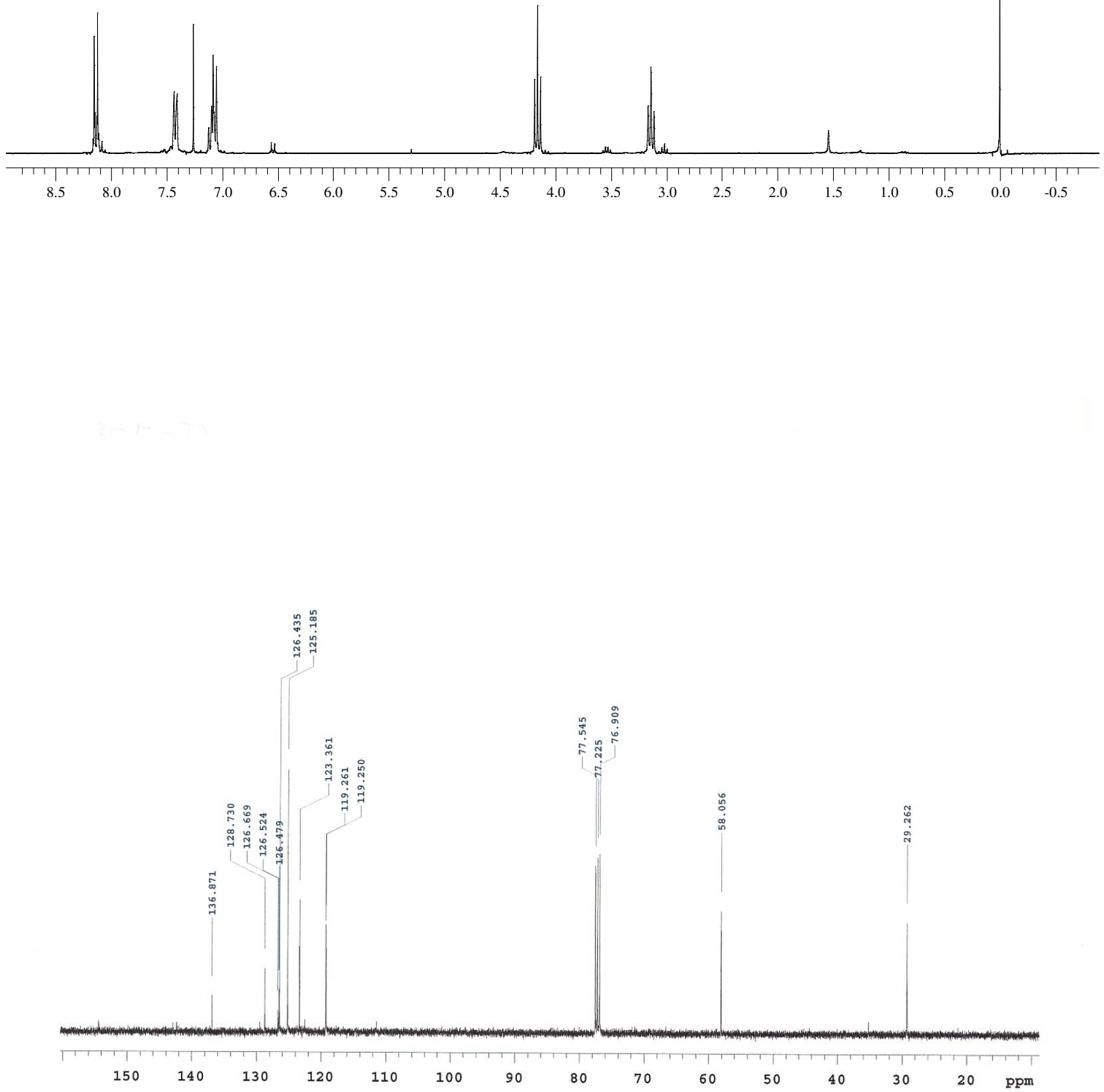


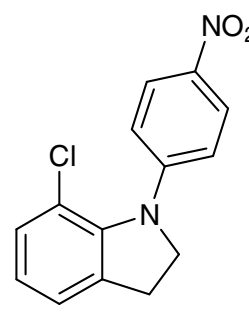

(2i)
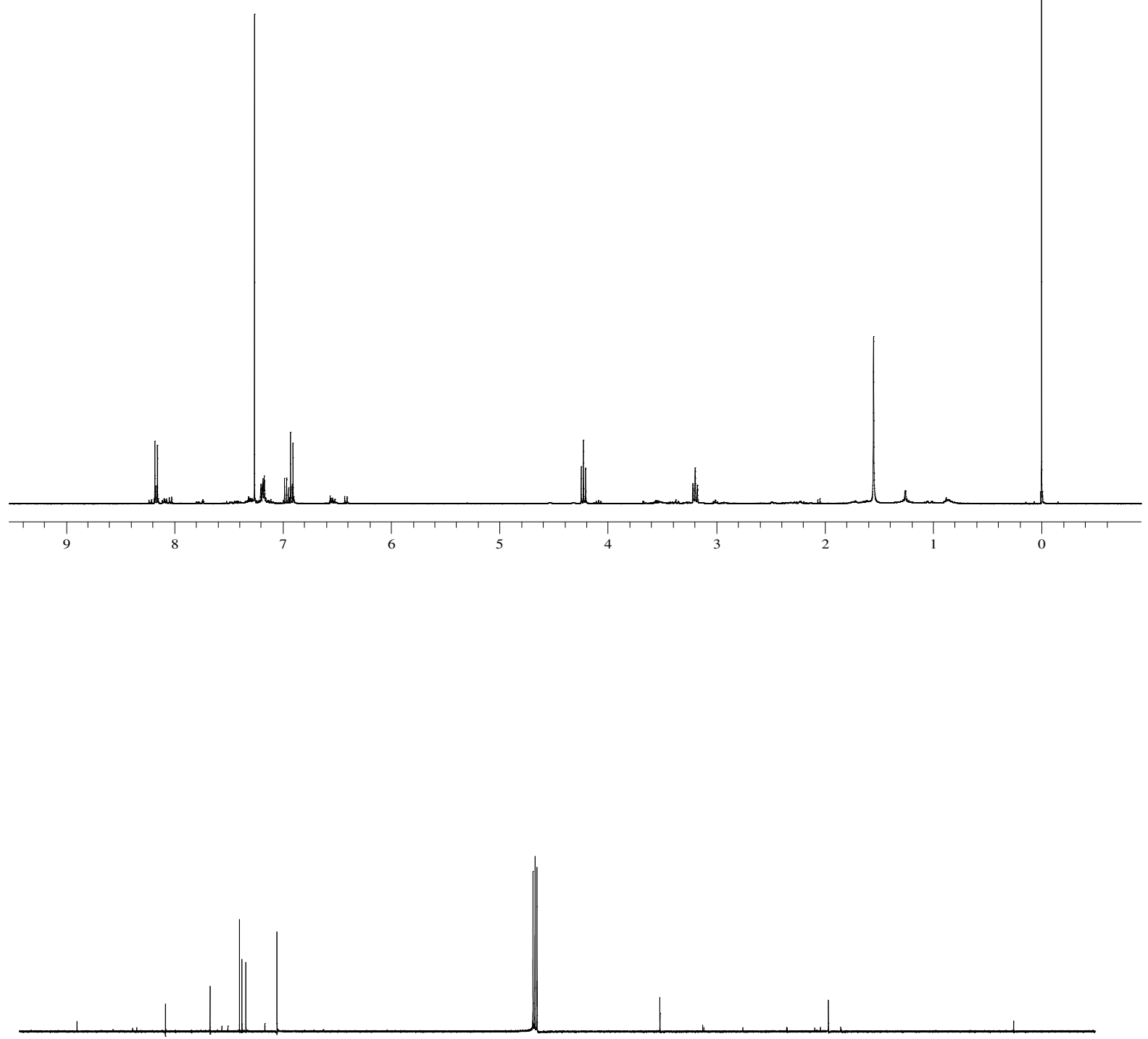

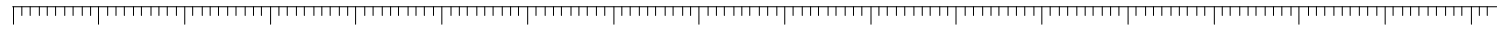

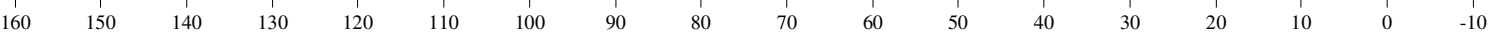



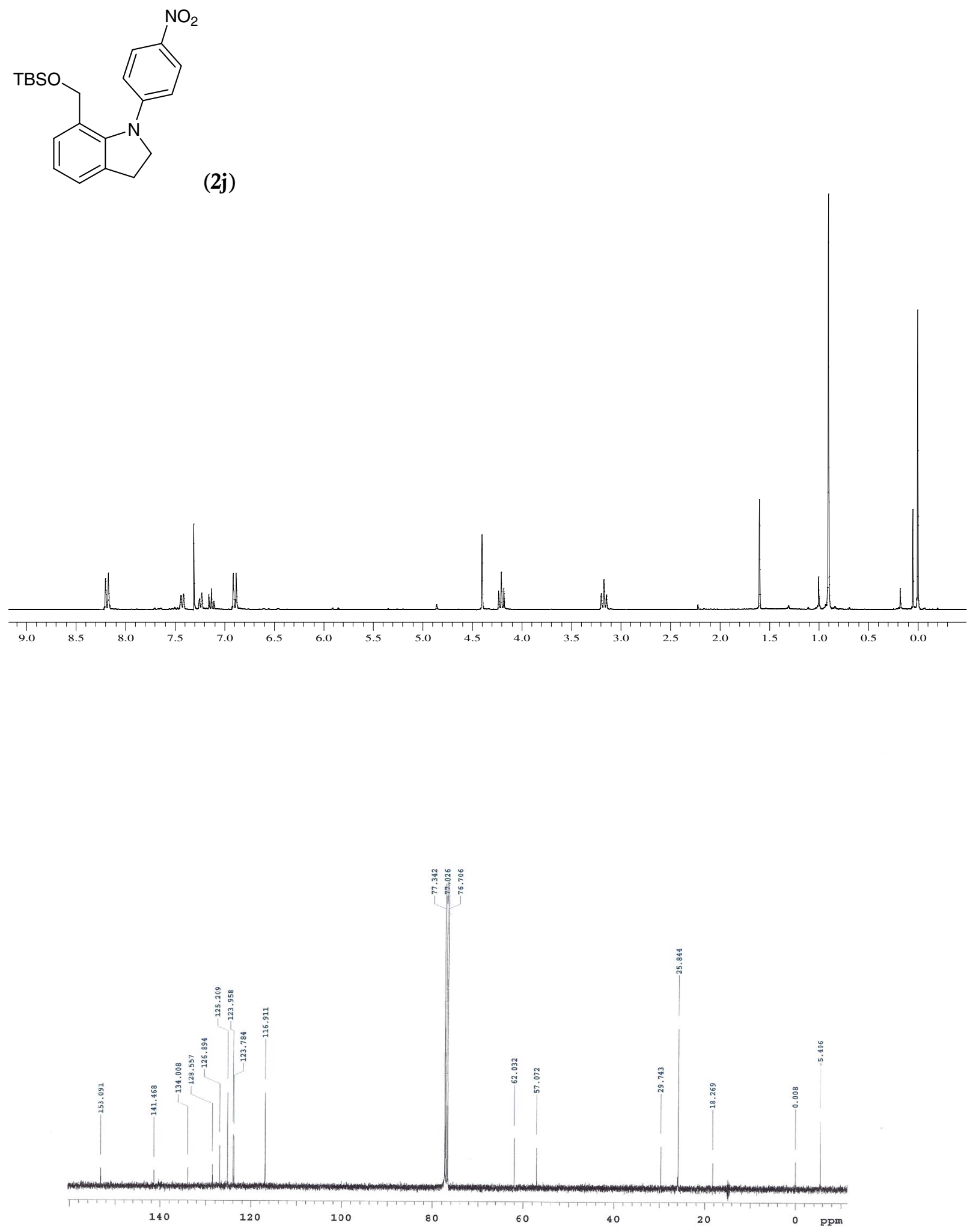
<smiles>O=[N+]([O-])c1ccc(N2CCc3ccc4ccccc4c32)cc1</smiles>
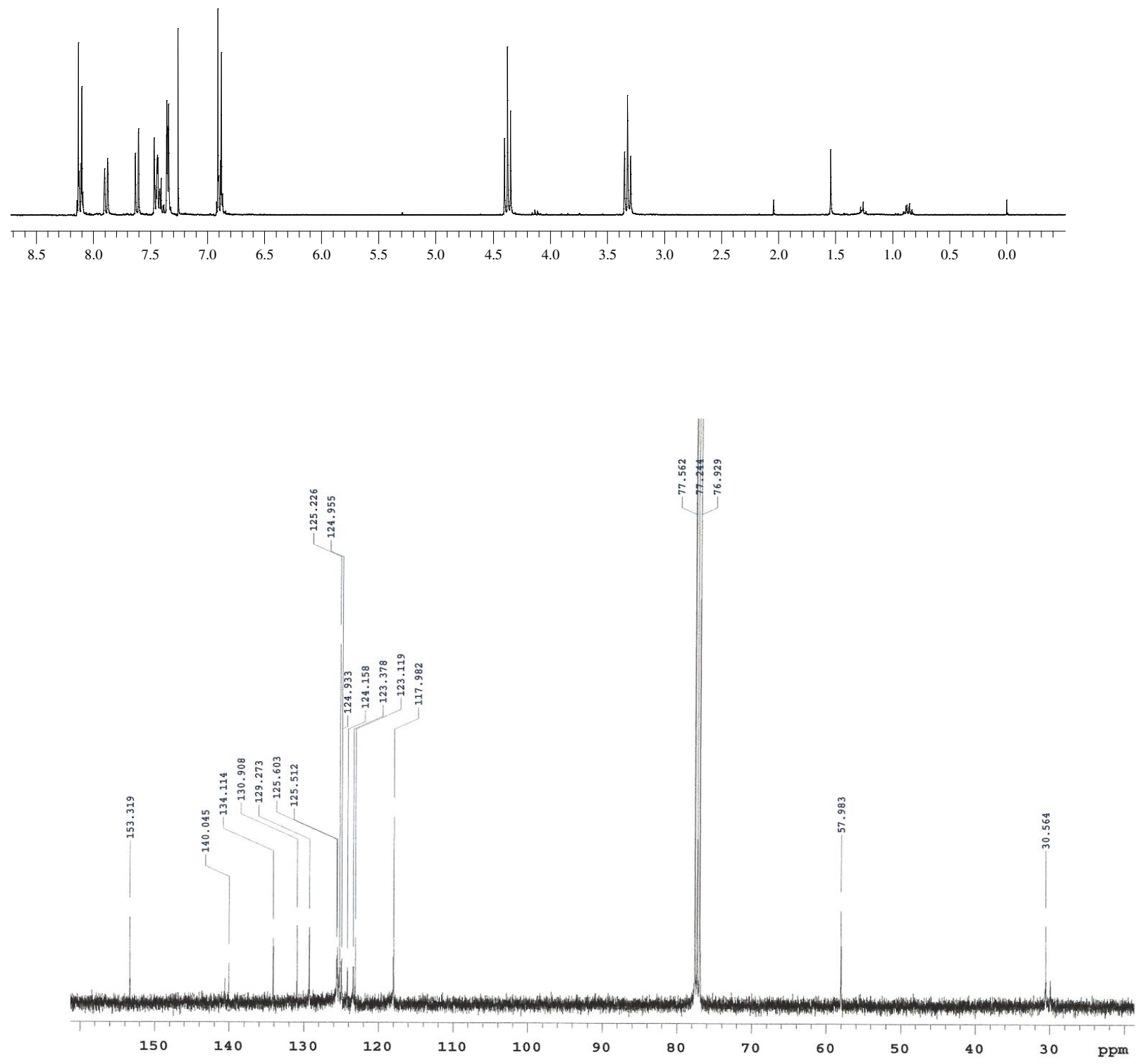

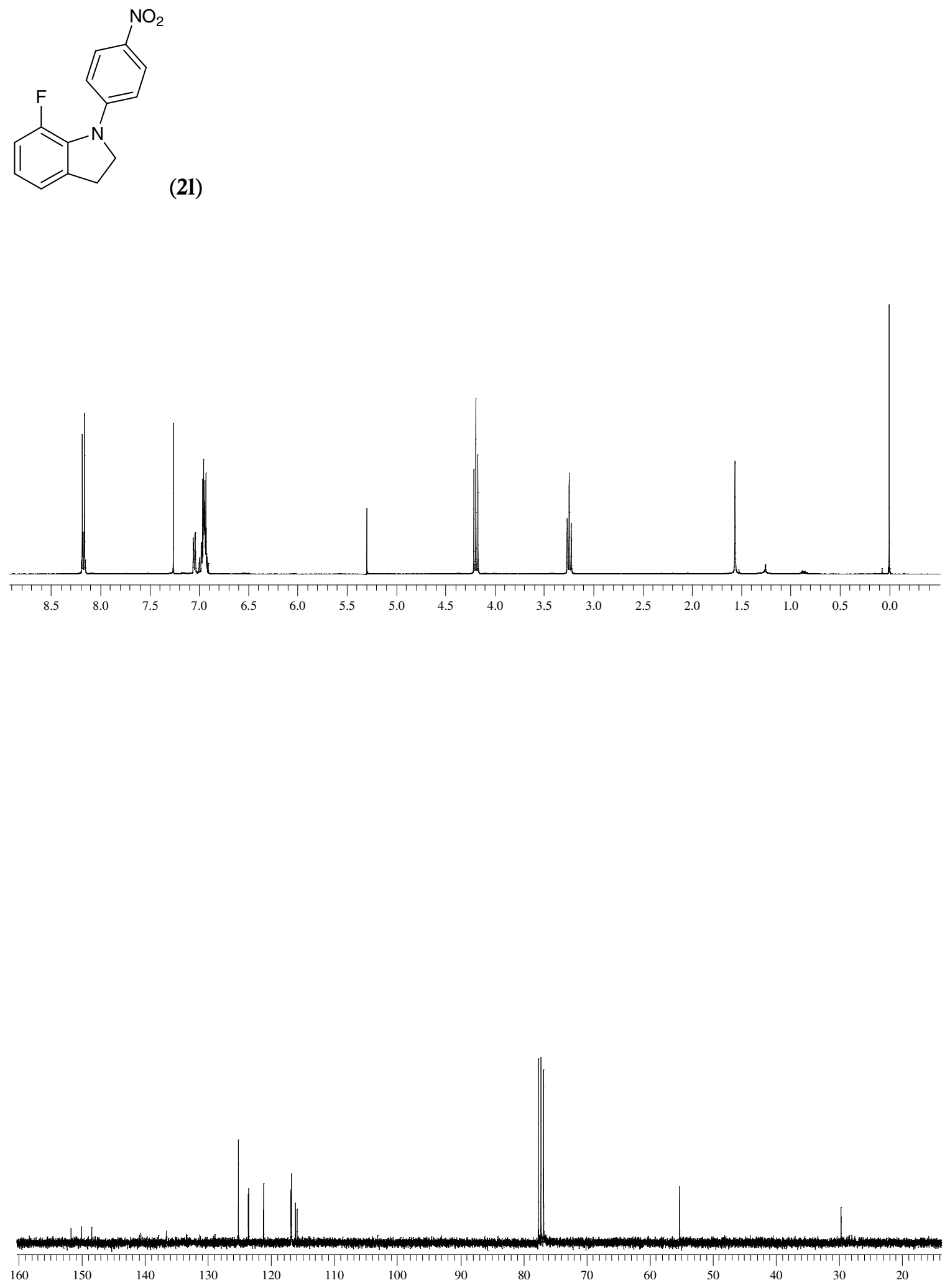


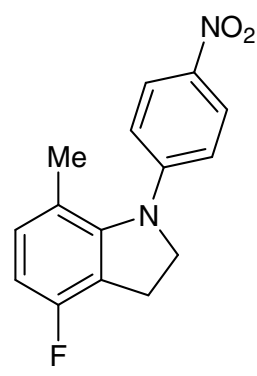

(2m)
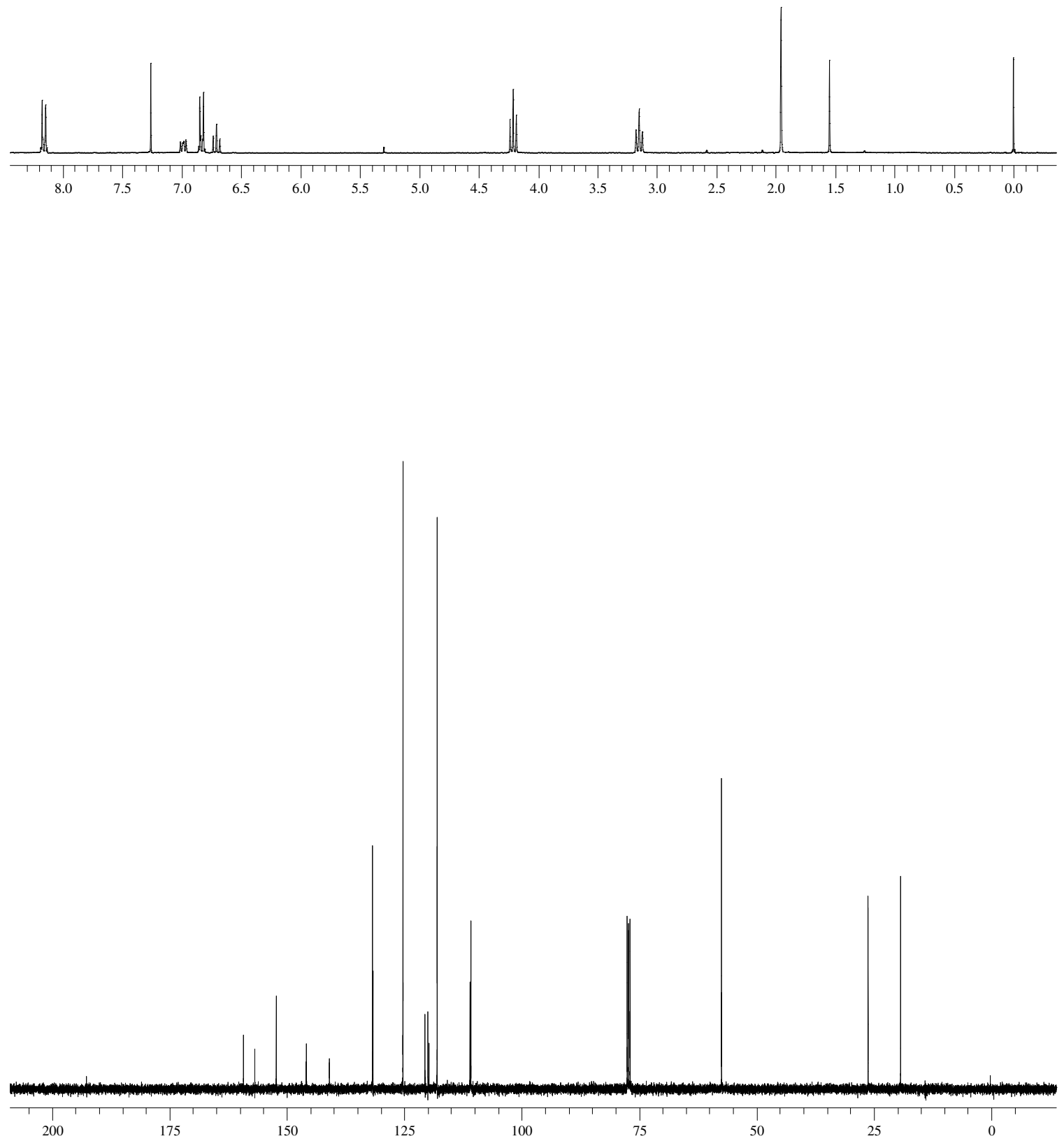

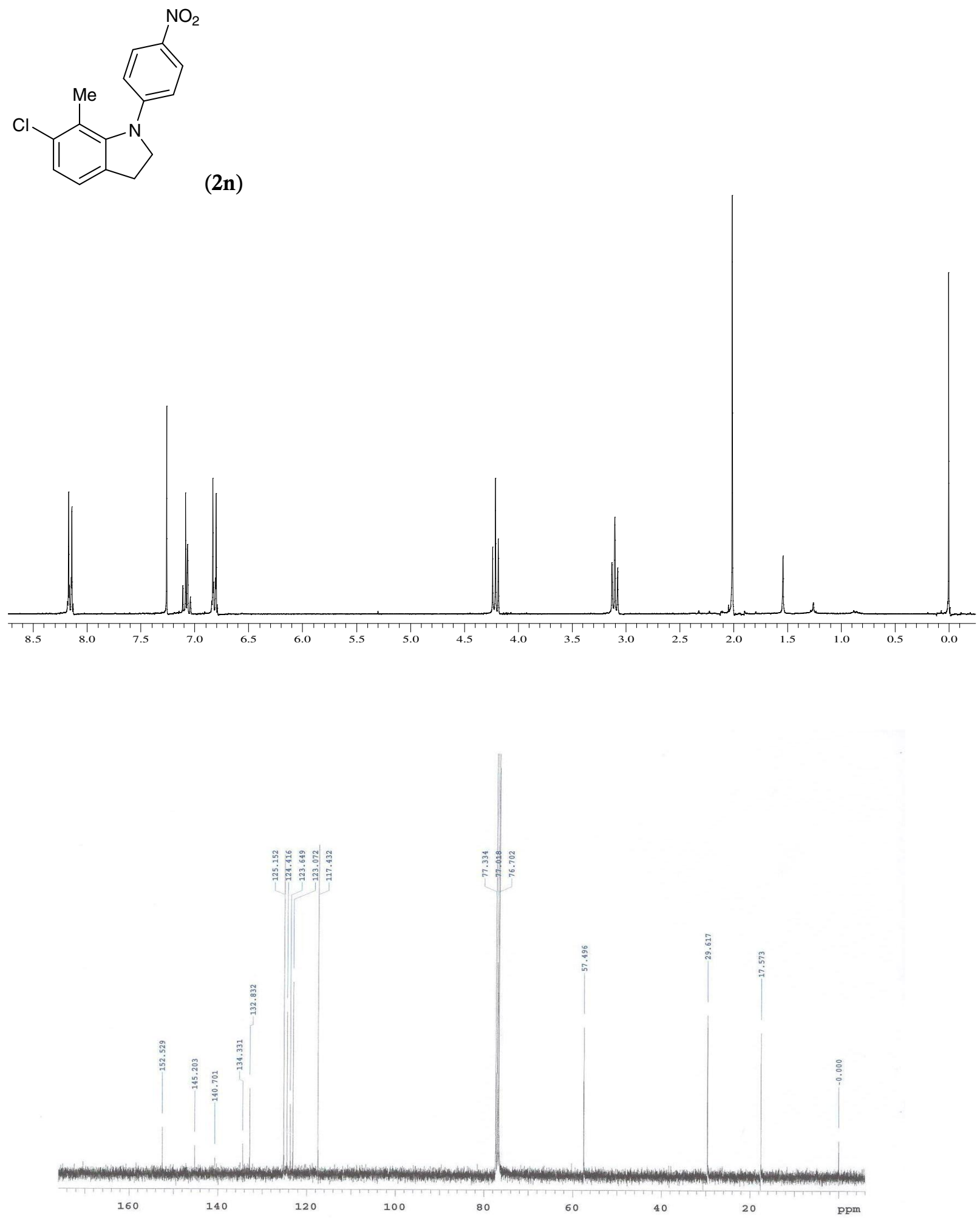


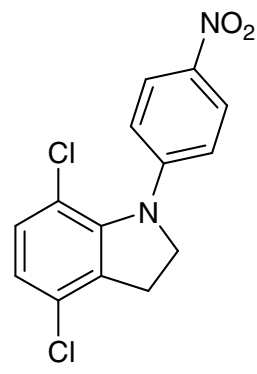

(2o)
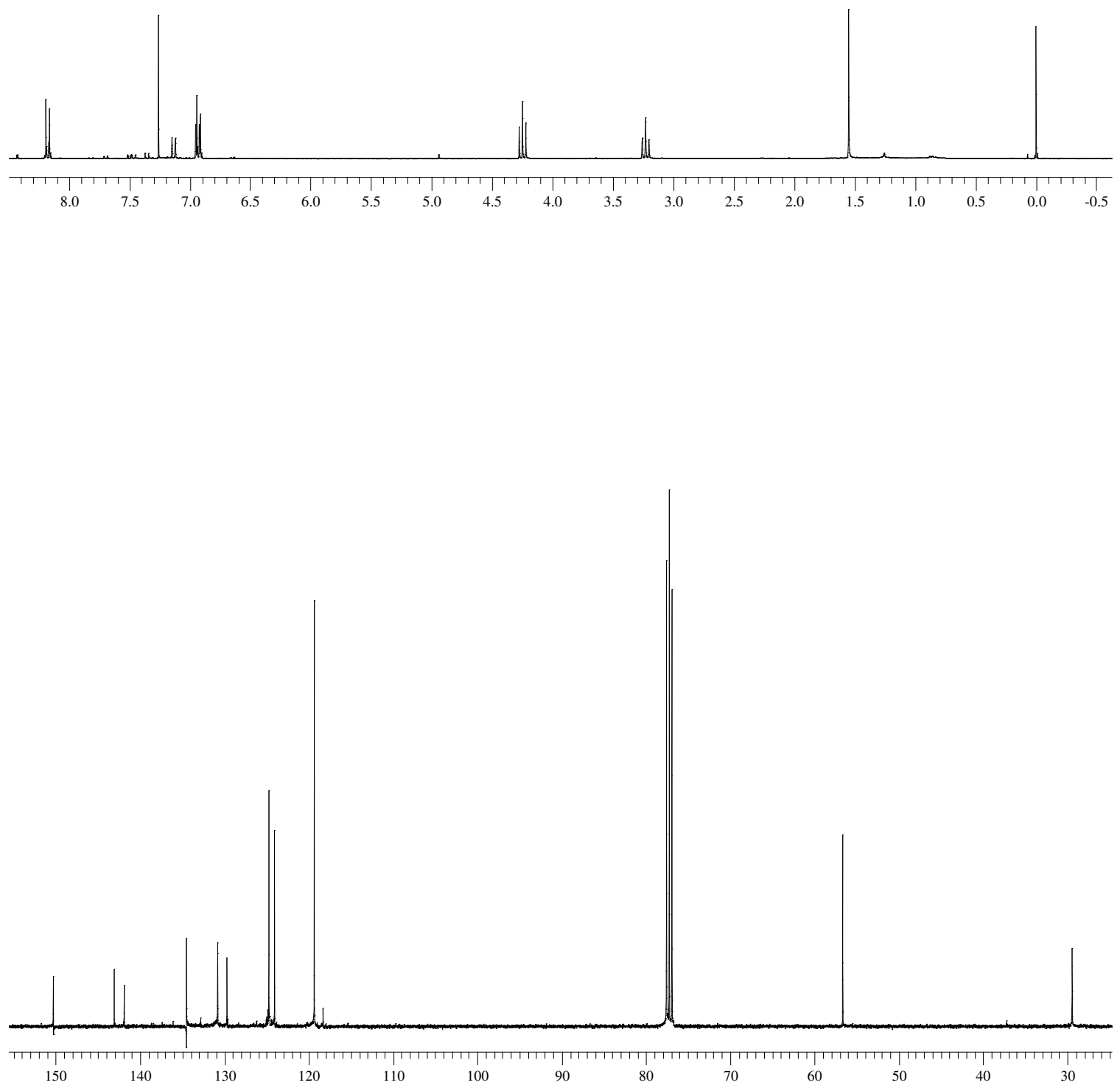

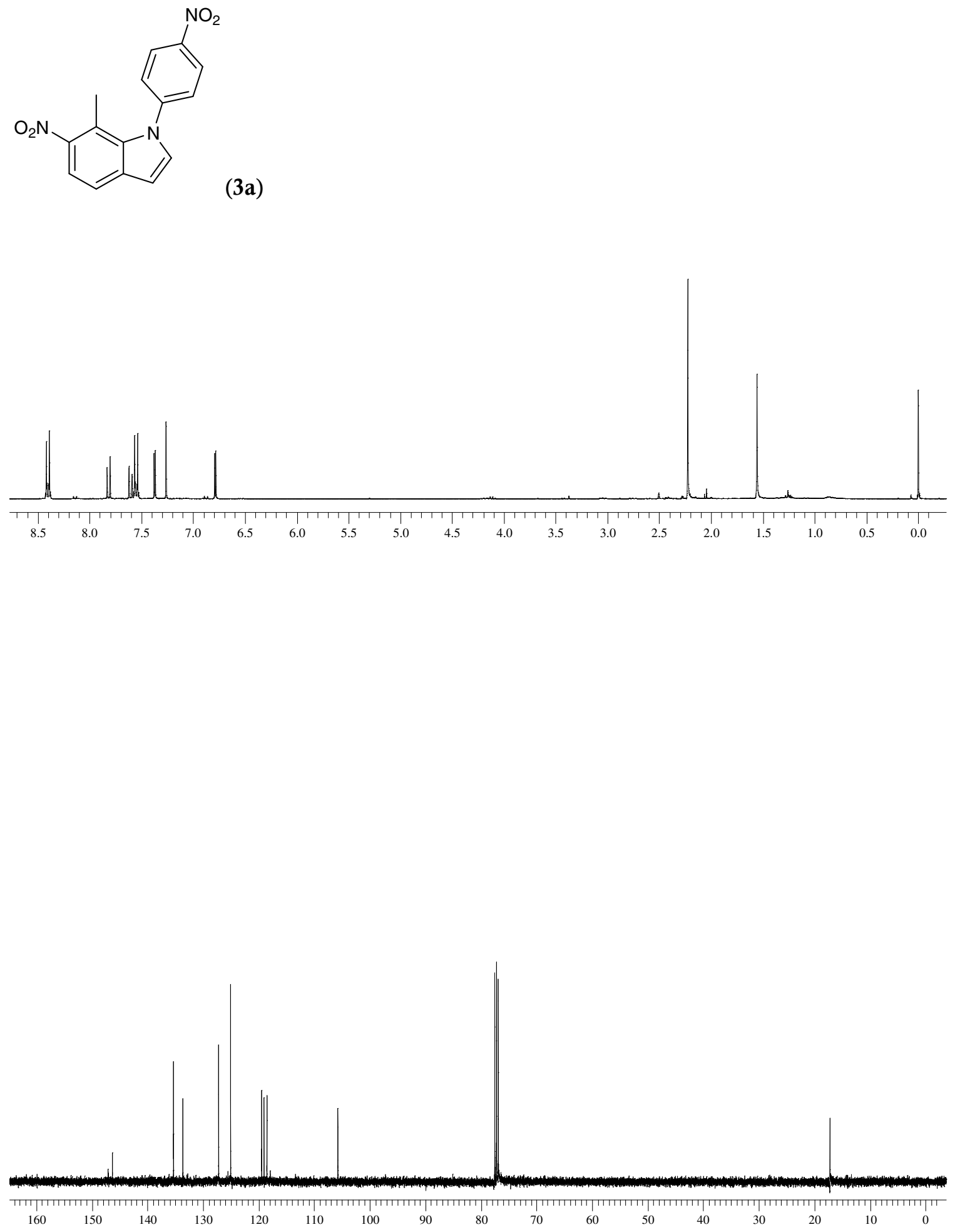


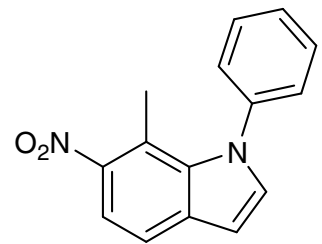

(3b)
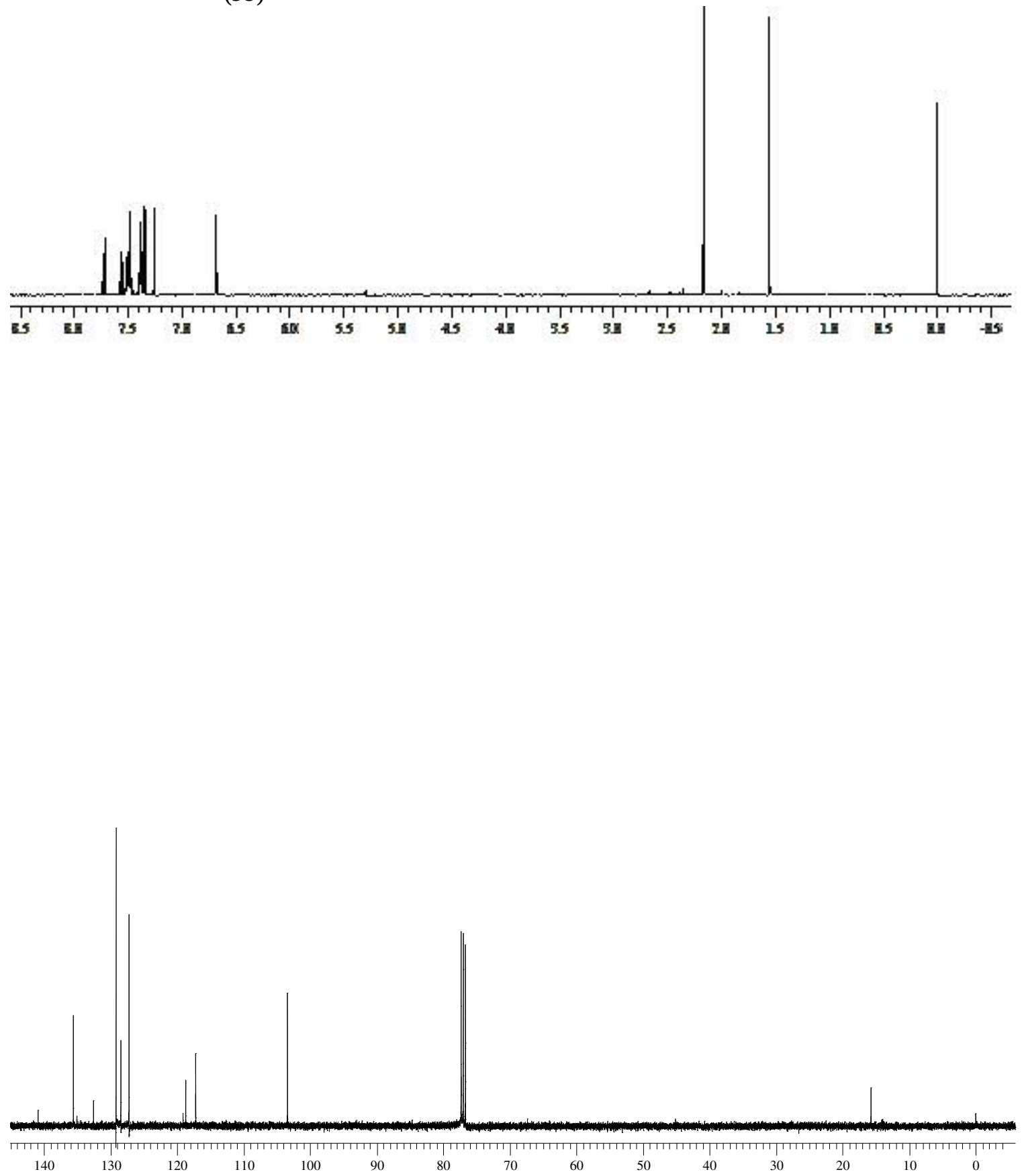
<smiles>CCOC(=O)N1CCc2cccc(C)c21</smiles>

(2d)

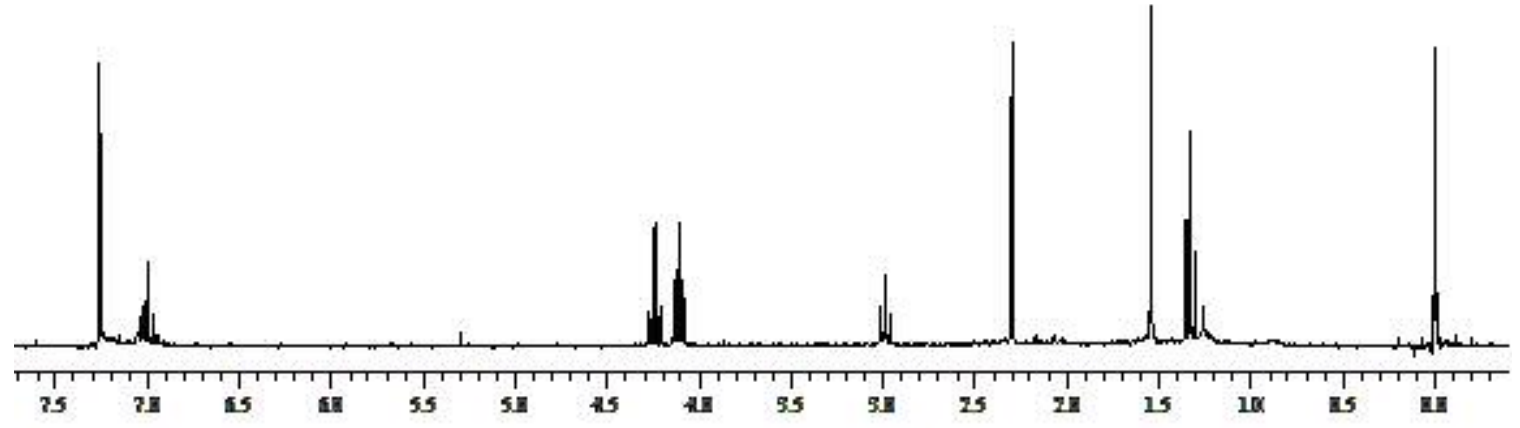

File: 20070906-PT_5_39_Carbon-001

Pulse Sequence: 32pul

Solvent: cdc13
Temp. $25.0 \mathrm{C} / 298.1 \mathrm{~K}$

Operator: mfleming

Relax. delay $0.200 \mathrm{sec}$

Pulse 45.0 degrees

Acq. time $1.337 \mathrm{~s}$
Width $24509.8 \mathrm{~Hz}$

20000 repetition

DECOUPLE H1, 399.7500357 MH

Power $40 \mathrm{~dB}$

continuously on
WALTZ-16 modulate

DATA PROCBSSING

Line broadening 0.5 Hz

.
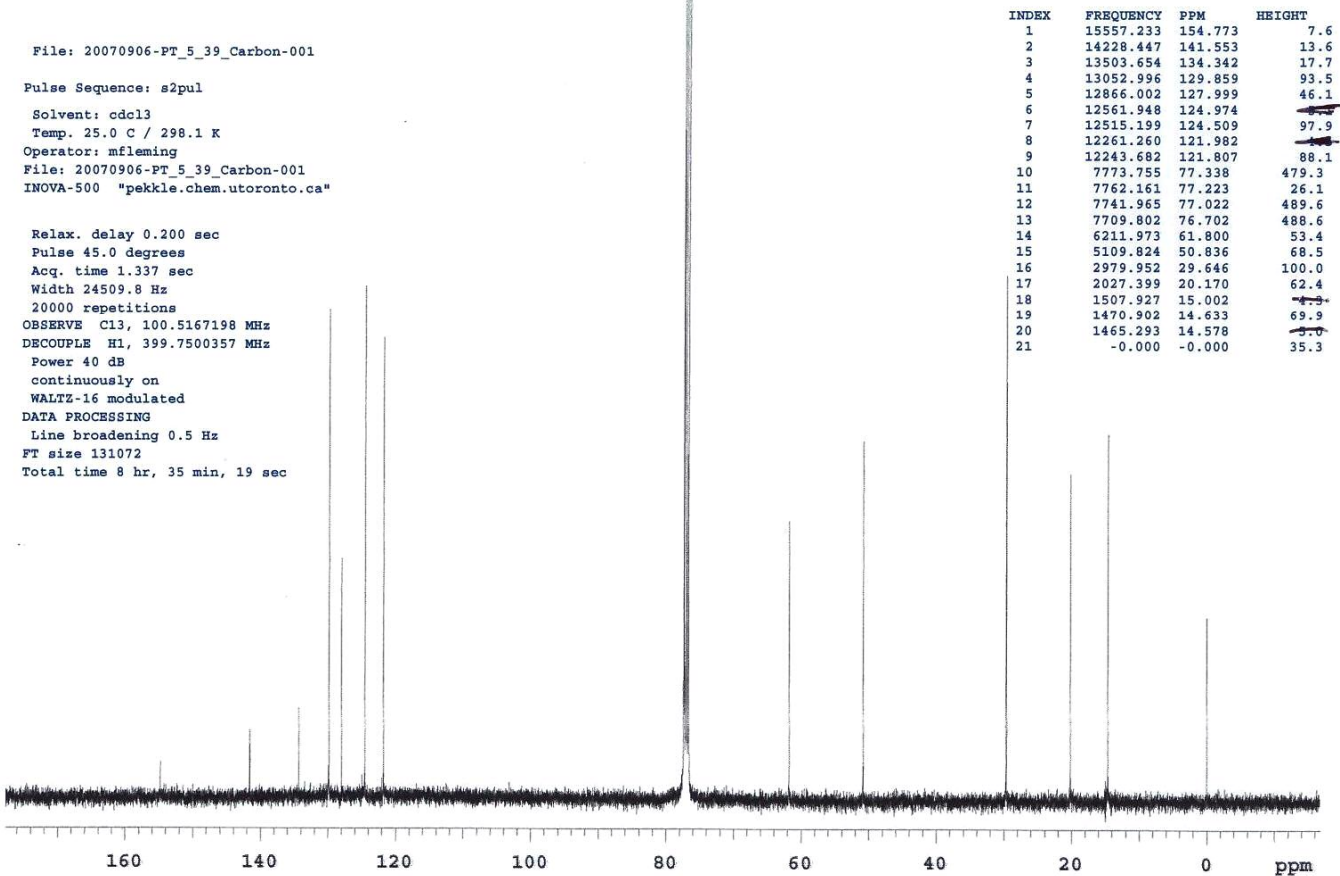

160

20

ppm 


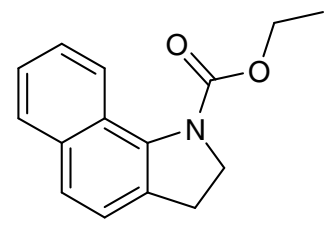

(2r)
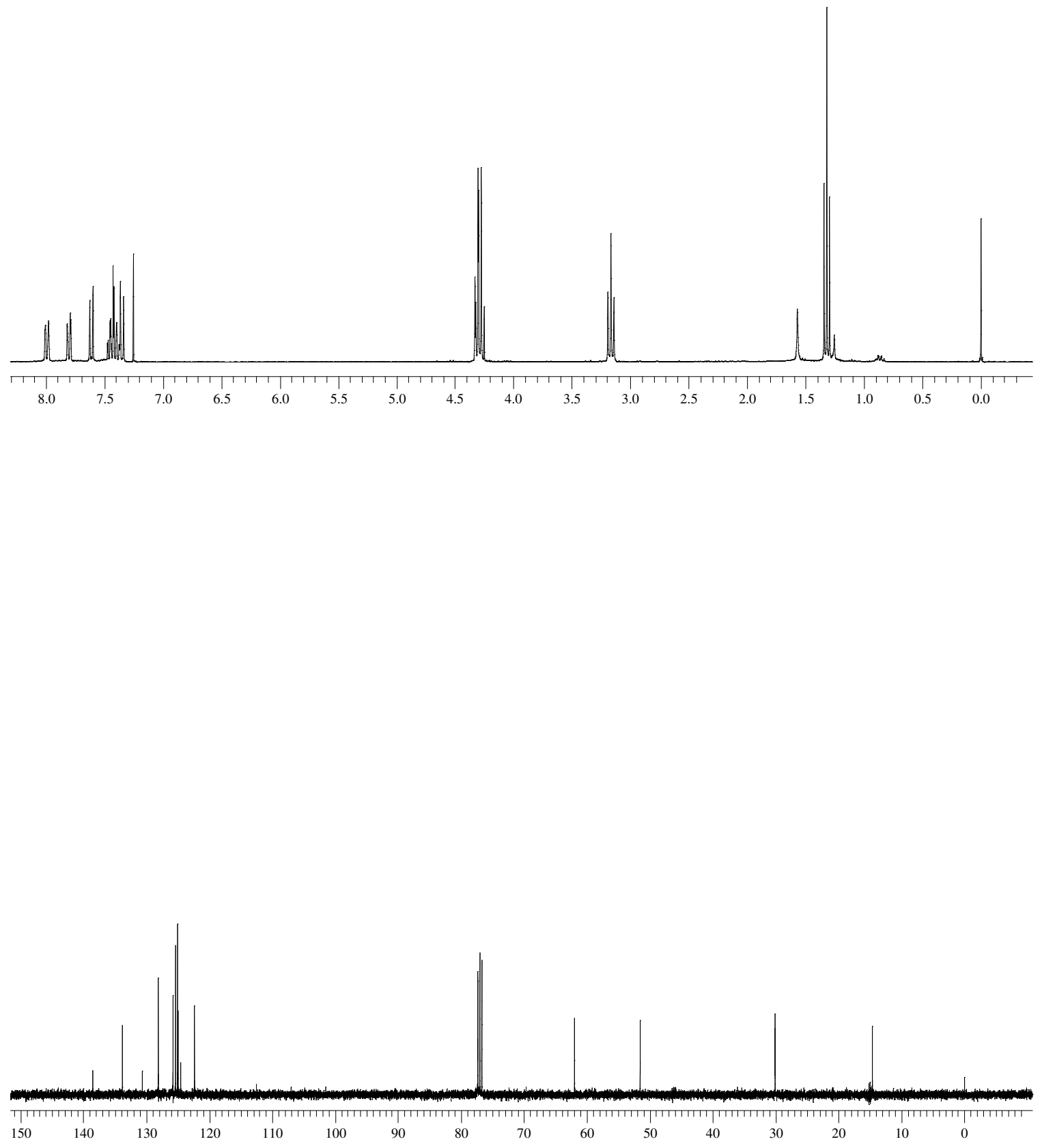


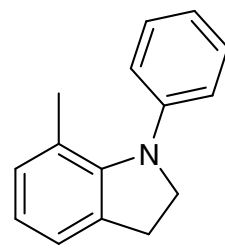

(2e)
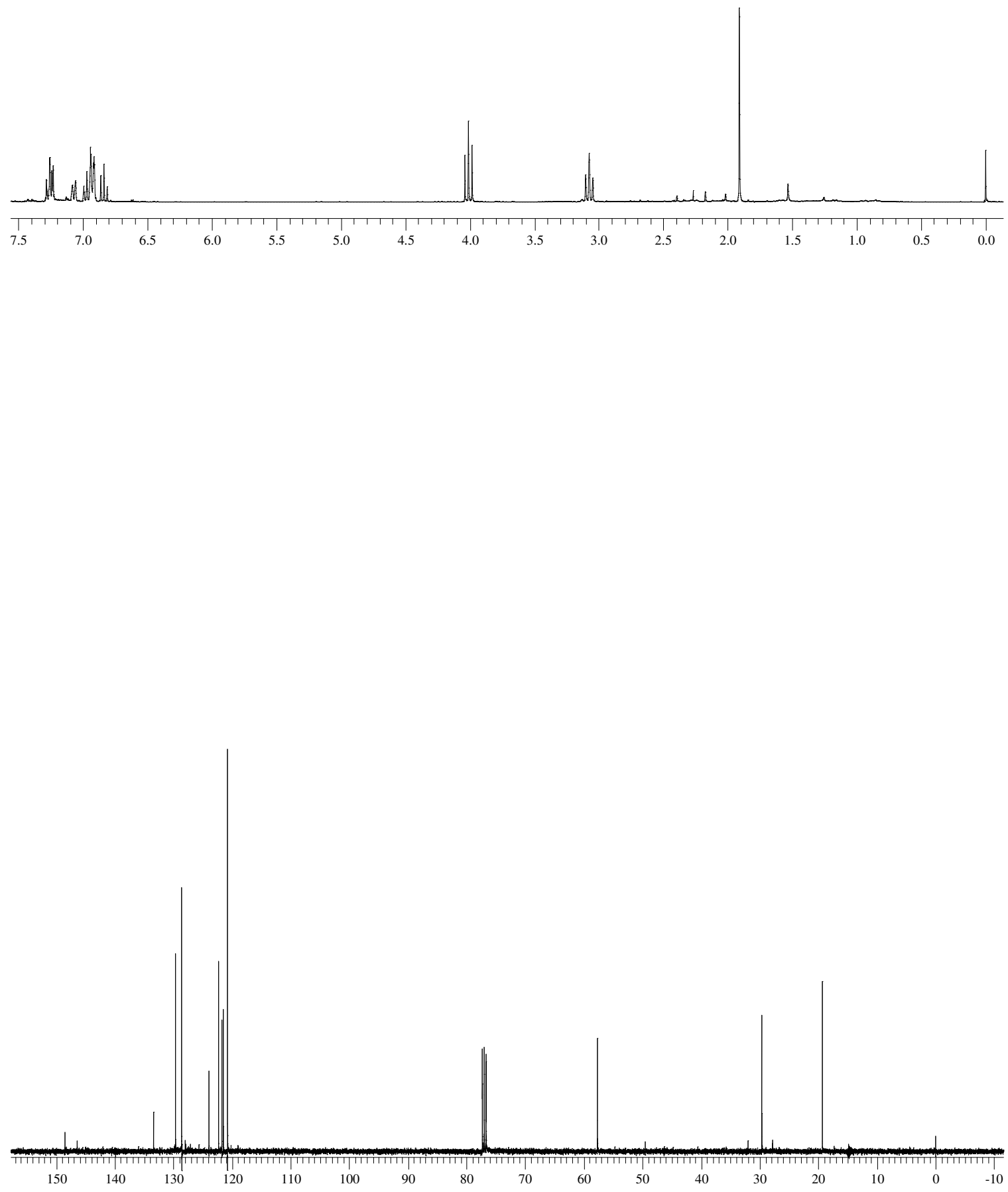
<smiles>c1ccc(N2CCc3ccc4ccccc4c32)cc1</smiles>

(2s)
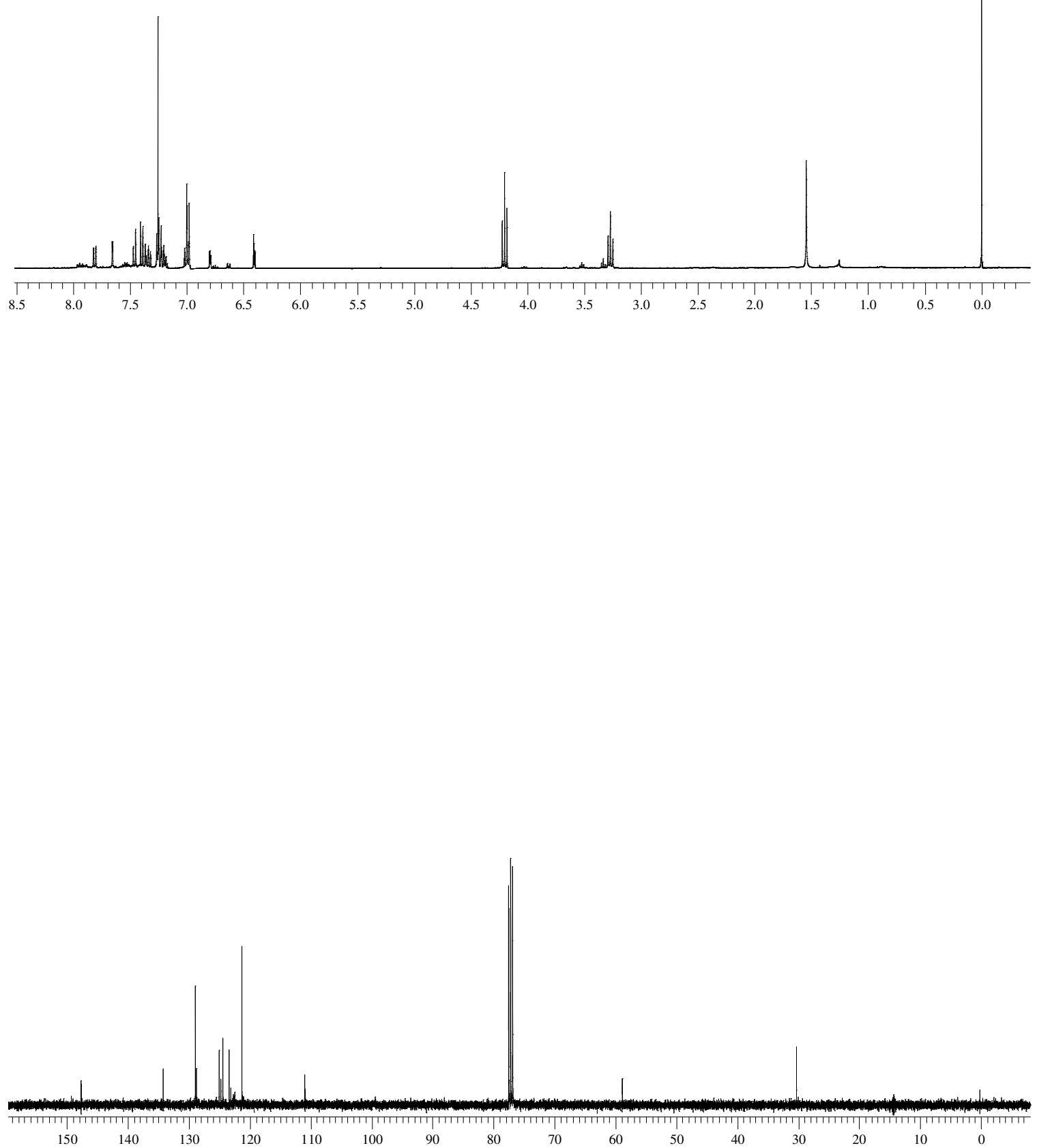

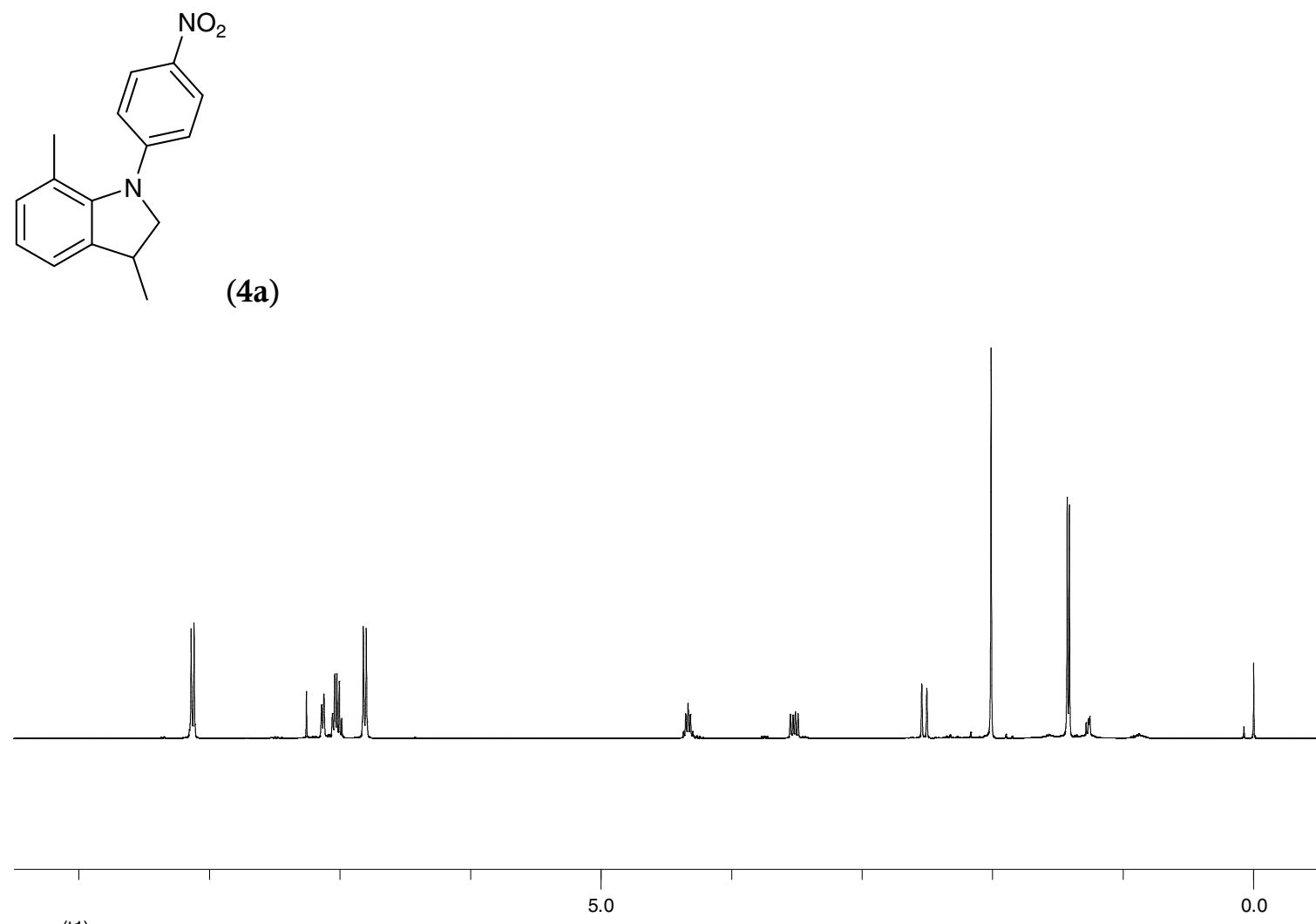

ppm (t1)
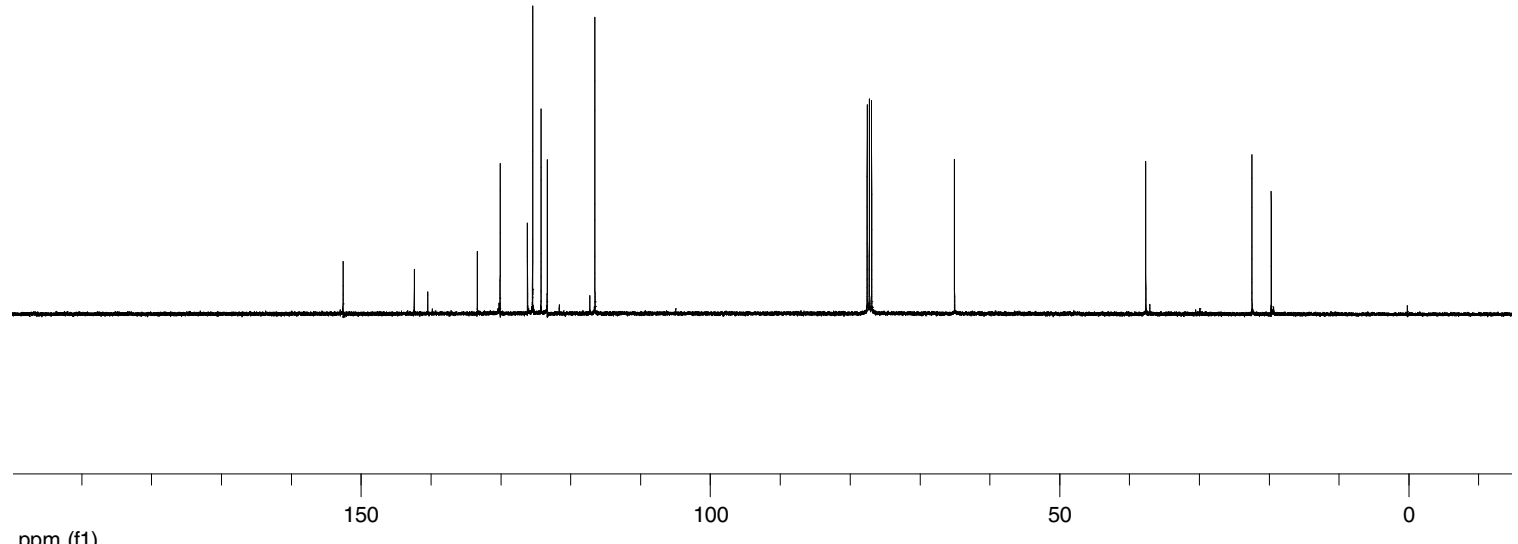

ppm (f1) 


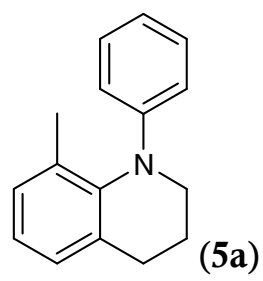
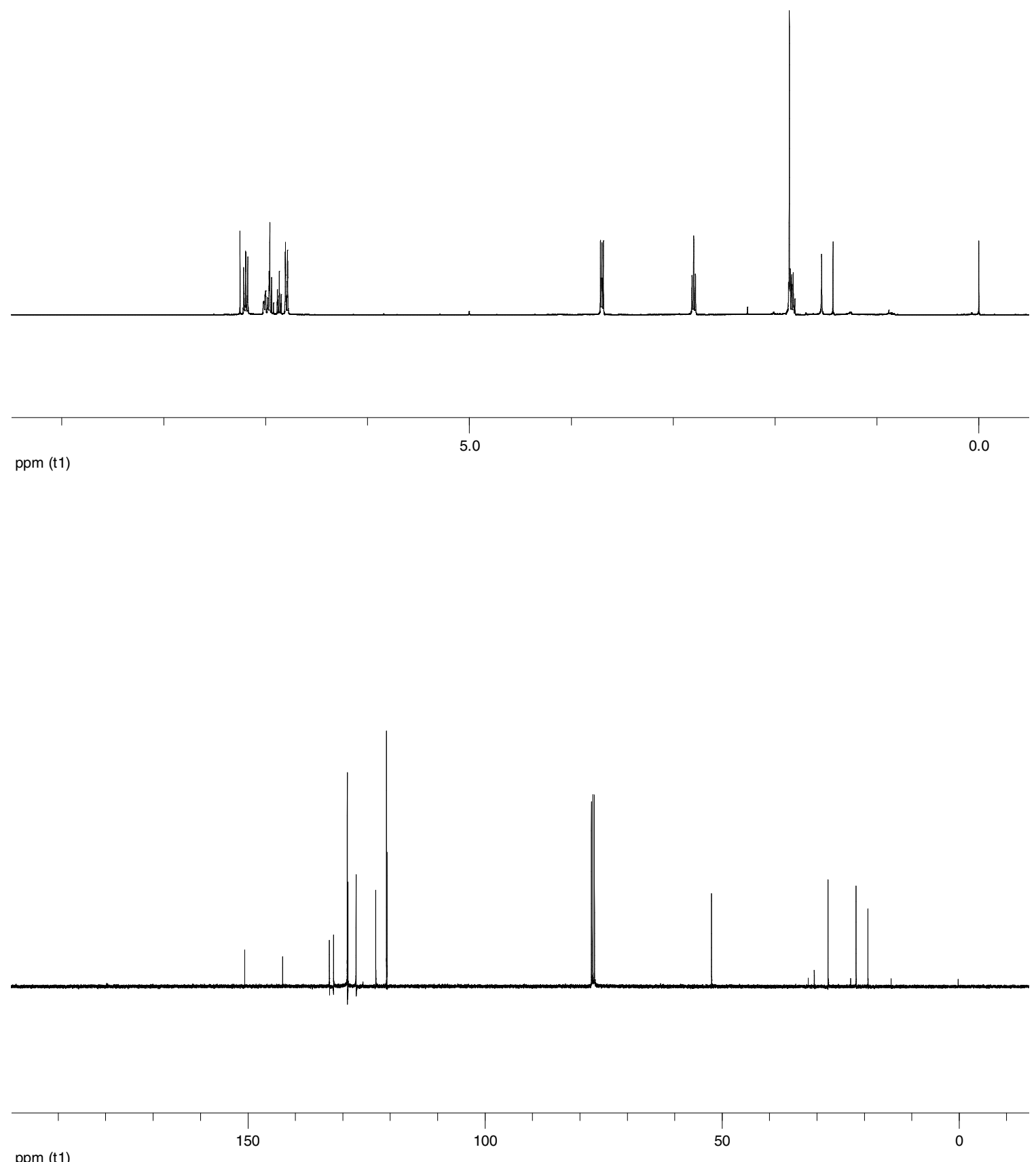

ppm (t1) 
<smiles></smiles>
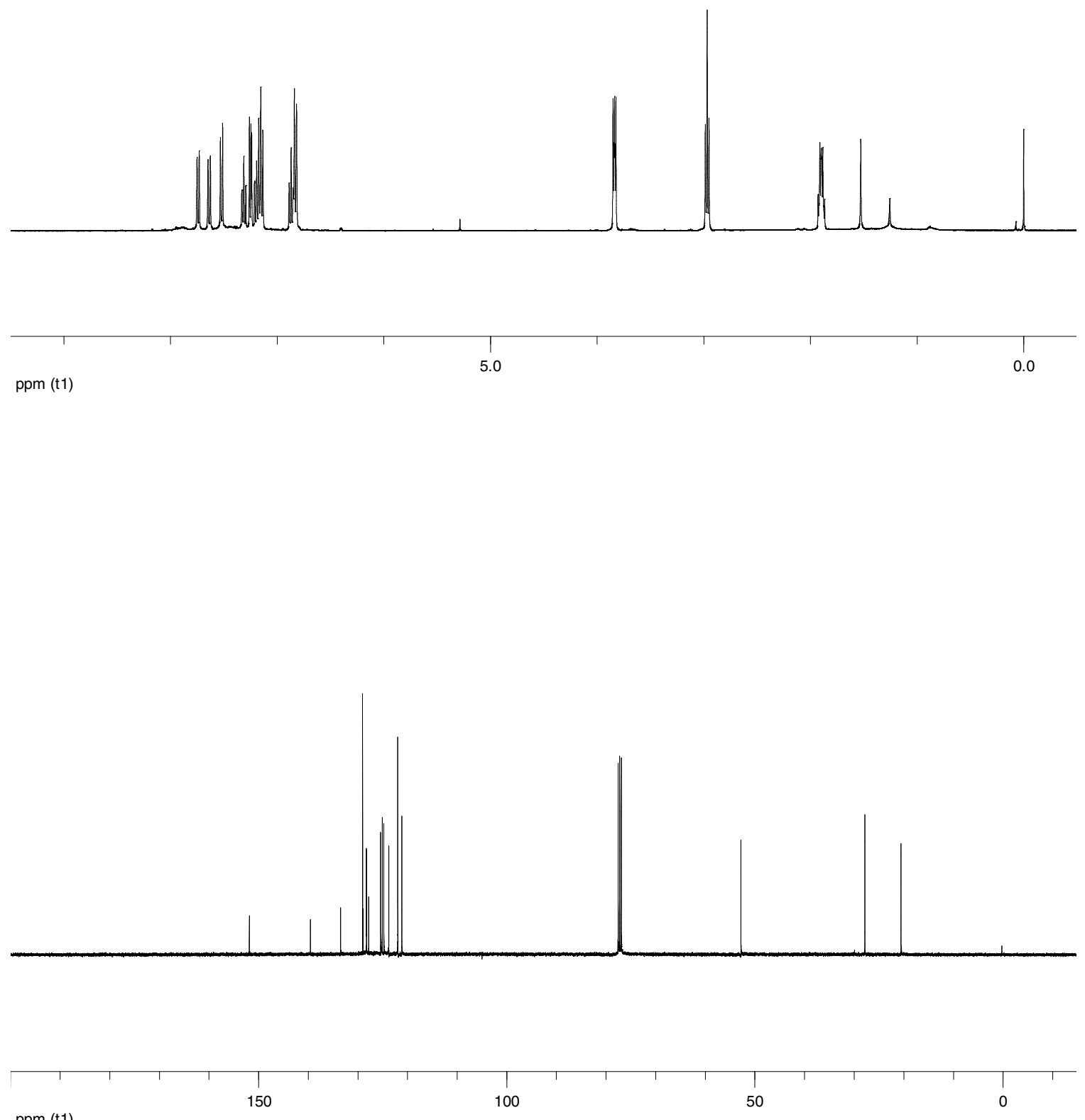

ppm (t1)

X-ray crystal structure for $\mathbf{2 f}$ 


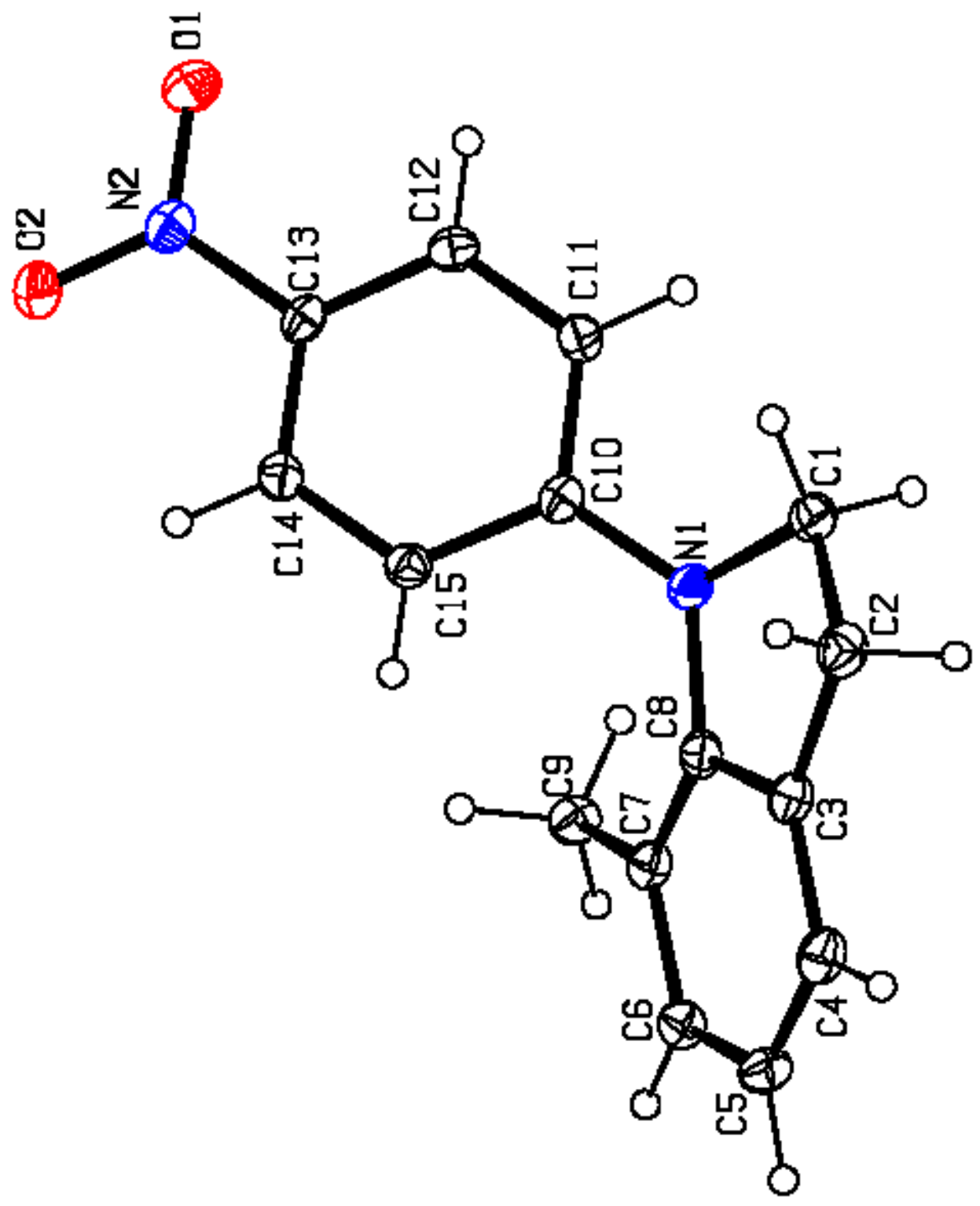

